\title{
The exterior Bitsadze-Lavrentjev problem for quaterelliptic-quaterhyperbolic equations in a doubly connected domain
}

\author{
John Michael Rassias
}

The National and Capodistrian University of Athens, Pedagogical Department, Section of Mathematics and Informatics, 4, Agamemnonos Str., Aghia Paraskevi, Athens, Attikis 15342, Greece.

E-mail: jrass@otenet.gr, jrassias@primedu.uoa.gr, Ioannis.Rassias@primedu.uoa.gr

\begin{abstract}
The famous Tricomi equation was established in 1923, by F. G. Tricomi, who is the pioneer of parabolic elliptic and hyperbolic boundary value problems and related problems of variable type. In 1945, F. I. Frankl established a generalization of these problems for the well-known Chaplygin equation. In 1953 and 1955, M. H. Protter generalized these problems even further. In 1977, we generalized these results in several n-dimensional simply connected domains. In 1950-1951, M. A. Lavrentjev and A. V. Bitsadze investigated the Bitsadze - Lavrentjev equation. In 1990, we proposed the exterior Tricomi problem. In 2002, we considered uniqueness of quasi-regular solutions for a bi-parabolic elliptic bi-hyperbolic Tricomi problem. In 2006, G. C. Wen investigated the exterior Tricomi problem for general mixed type equations. In 2011, we established the exterior Tricomi and Frankl problems for quaterelliptic - quaterhyperbolic equations. In 2014, D. Amanov and J. M. Rassias investigated boundary value problems for the higher order generalized mixed-parabolic equation. In this paper we investigate the exterior Bitsadze-Lavrentjev problem for quaterelliptic -quaterhyperbolic Bitsadze-Lavrentjev PDEquations with eight parabolic lines in a doubly connected domain and propose open problems. These problems are of vital importance in fluid mechanics.
\end{abstract}

2010 Mathematics Subject Classification. 35MO5.

Keywords. Quasi-regular solution, Bitsadze-Lavrentjev PDEquation, quaterelliptic equation, quaterhyperbolic equation, Bitsadze-Lavrentjev problem..

\section{Introduction}

In 1904, S. A. Chaplygin [11] pointed out that the nonlinear equation of an adiabatic potential perfect gas :

$$
\left(\rho^{2} \alpha^{2}-\psi_{y}^{2}\right) \psi_{x x}+2 \psi_{x} \psi_{y} \psi_{x y}+\left(\rho^{2} \alpha^{2}-\psi_{x}^{2}\right) \psi_{y y}=0
$$

is closely connected with the study of the linear mixed type equation

$$
K(y) u_{x x}+u_{y y}=0,
$$

named Chaplygin equation, where $\psi=\psi(x, y)$ is the stream function, $\alpha=$ local velocity of sound and $\rho=$ density of gas.

In 1923, F. G. Tricomi [19] initiated the work on boundary value problems for linear partial differential mixed type equations of second order and related equations of variable type. The well-known mixed type partial differential equation is called Tricomi equation :

$$
y u_{x x}+u_{y y}=0
$$


after F. G. Tricomi , who introduced this equation, for functions $u=u(x, y)$ in a real $(x, y)$-region. It plays a central role in the mathematical analysis of the transonic flows, as it is of elliptic and hyperbolic type, where the coefficient $y$ of the second order partial derivative of the involved function $u=u(x, y)$ with respect to $x$, changes sign. Besides this equation is of parabolic type, where $y$ vanishes. In 1945, F. I. Frankl [3] drew attention to the fact that the Tricomi problem was closely connected to the study of gas flow with nearly sonic speeds. In 1953 and 1955, M. H. Protter [7] generalized and improved the afore-mentioned results in the euclidean plane. In 1977, we [8] generalized these results in $R^{n},(n>2)$. In 1950-1951, M. A. Lavrentjev and A. V. Bitsadze ([29]-[30]) investigated the famous Bitsadze-Lavrentjev equation:

$$
\operatorname{sgn}(y) u_{x x}+u_{y y}=0
$$

where

$$
\operatorname{sgn}(y)=1, y>0 ;=0, y=0 ;=-1, y<0 .
$$

In 1982, we [9] established a maximum principle of the Cauchy problem for hyperbolic equations in $R^{n+1},(n \geq 2)$. In 1983, we [10] solved the Tricomi problem with two parabolic lines of degeneracy and, in 1992, we [12] established the well-posedness of the Tricomi problem in euclidean regions. Interesting results for the Tricomi problem were achieved by G. Baranchev [1] in 1986, and M. Kracht and E. Kreyszig [4] in 1986, as well. Related information was reported by G. Fichera [2] in 1985, and E. Kreyszig ([5]-[6]) in 1989 and 1994. Our ([11], [14]-[15]) work, in 1990 and 1999, was in similar areas of mixed type equations. In 1990-2009, G. C. Wen et al. ([17], [20]-[28], [31]) have applied the complex analytic method and achieved fundamental uniqueness and existence results for solutions of the Tricomi and Frankl problems for the classical mixed type partial differential equations with boundary conditions. In 1993, R.I. Semerdjieva [18] introduced the hyperbolic equation

$$
K_{1}(y) u_{x x}+\left(K_{2}(y) u_{y}\right)_{y}+r u=f
$$

in the lower half-plane. In 1997, we [13] considered the more general case of the above hyperbolic equation, so that it was elliptic in the upper half-plane and parabolic on the line $y=0$. In 2002, we [16] considered the more general Tricomi problem with partial differential equation the new bi-parabolic elliptic bi-hyperbolic equation

$$
L u=K_{1}(y)\left(M_{2}(x) u_{x}\right)_{x}+M_{1}(x)\left(K_{2}(y) u_{y}\right)_{y}+r(x, y) u=f(x, y),
$$

which is parabolic on both segments $x=0,0<y \leq 1 ; y=0,0<x \leq 1$, elliptic in the euclidean region $G_{e}=\left\{(x, y) \in G\left(\subset R^{2}\right): x>0, y>0\right\}$ and hyperbolic in both regions

$$
G_{h_{1}}=\left\{(x, y) \in G\left(\subset R^{2}\right): x>0, y<0\right\} ; G_{h_{2}}=\left\{(x, y) \in G\left(\subset R^{2}\right): x<0, y>0\right\},
$$

with $G$ the mixed domain of (1.1). In 1999, we [15] proved existence of weak solutions for a particular Tricomi problem. Then we established uniqueness of quasi-regular solutions ( [8], [10][13], [16]) for the Tricomi problem. In 2011, we ([32]-[33]) established the uniqueness of quasi-regular solutions for the exterior Tricomi and Frankl problems. In this paper we investigate the exterior Bitsadze-Lavrentjev problem for general Bitsadze-Lavrentjev quaterelliptic and quaterhyperbolic equations:

$$
L u=\operatorname{sgn}(y(y-1)|k(y)|) u_{x x}+\operatorname{sgn}(x(x+1)|m(x)|) u_{y y}+r(x, y) u=f(x, y),
$$




$$
k=k(y) \neq 0 ; m=m(x) \neq 0,
$$

everywhere in $D$, with eight parabolic lines of degeneracy in a doubly connected mixed domain and establish uniqueness of quasi-regular solutions. Also we propose new open problems. These results are interesting in Aerodynamics and Hydrodynamics. The Mixed type partial differential equations are encountered in the theory of transonic flow and they give rise to special boundary value problems, called the Tricomi and Frankl as well as Bitsadze-Lavrentjev problems. The Transonic flows involve a transition from the subsonic to the supersonic region through the sonic.

Definition 1.1. The Bitsadze-Lavrentjev problem or Problem BL consists of finding a function $u$ which satisfies the afore-mentioned Bitsadze-Lavrentjev equation (1.2) in a mixed domain $D$ :

a simply connected and bounded $(x, y)$-region by a rectifiable Jordan (non-self-intersecting) elliptic $\operatorname{arc} \sigma$ (for $y>0$ ) with endpoints $O=(0,0)$ and $A=(1,0)$ and by two hyperbolic characteristics $\Gamma, \gamma$ of the Bitsadze-Lavrentjev equation satisfying the pertinent characteristic equation such that these characteristics $\Gamma, \gamma$ meet at a point $P$ (for $y<0$ ) with $\Gamma$ emanating from $A=(1,0)$ and $\gamma$ from $O=(0,0)$,

$$
\Gamma: y=x-1 \text { and } \gamma: y=-x,
$$

and $u$ assumes prescribed continuous boundary values on both $\operatorname{arcs} \sigma$ and $\gamma$. The portion of $D$ lying in the upper half-plane, above the $x$-axis, is the elliptic region; portion of $D$ lying in the lower half-plane, below the $x$-axis, is the hyperbolic region; and the segment $O A$ is parabolic.

Definition 1.2. A function $u=u(x, y)$ is regular solution of Problem $B L$ if:

1. $\mathrm{u}$ is continuous in the closure of $D$ which is the union of $D$ with its boundary consisting of the three curves $\sigma, \Gamma, \gamma$;

2. The first order partial derivatives of $u$ are continuous in the closure of $D$ except points $O, A$, where they may have poles of order less than $2 / 3$;

3. The second order partial derivatives of $u$ are continuous in $D$ except possibly on $O A$ where they may not exist;

4. $u$ satisfies Bitsadze-Lavrentjev equation at all points of $D$ except $O A$;

5. $u$ assumes prescribed continuous boundary values on both $\operatorname{arcs} \sigma, \gamma$;

\section{Exterior Bitsadze-Lavrentjev problem: Tricomi Case}

Consider the general Bitsadze-Lavrentjev quaterelliptic - quaterhyperbolic equation (1.2) with eight parabolic lines of degeneracy in a bounded doubly connected mixed domain $D$ with a piecewise smooth boundary $\partial D$, where $f=f(x, y)$ is continuous in $D, r=r(x, y)$ is once-continuously differentiable in $D, K=K(y):=\operatorname{sgn}(y(y-1)|k(y)|)$ is discontinuous for $y \in\left[-k_{1}, k_{2}\right]$ with $-k_{1}=\inf \{y:(x, y) \in D\}$ and $k_{2}=\sup \{y:(x, y) \in D\}$, and $M=M(x):=\operatorname{sgn}(x(x+1)|m(x)|)$ is discontinuous for $x \in\left[-m_{1}, m_{2}\right]$ with $-m_{1}=\inf \{x:(x, y) \in D\}$ and $m_{2}=\sup \{x:(x, y) \in D\}$, so that $k=k(y) \neq 0 ; m=m(x) \neq 0$ everywhere in $D$. Besides

$$
K=\left\{\begin{array}{rll}
+1 & \text { for } & \{y<0\} \cup\{y>1\} \\
0 & \text { for } & \{y=0\} \cup\{y=1\} \\
-1 & \text { for } & \{0<y<1\}
\end{array} \quad ; \quad M=\left\{\begin{array}{rlll}
+1 & \text { for } & \{x<-1\} \cup\{x>0\} \\
0 & \text { for } & \{x=0\} \cup\{x=-1\} \\
-1 & \text { for } & \{-1<x<0\}
\end{array} .\right.\right.
$$




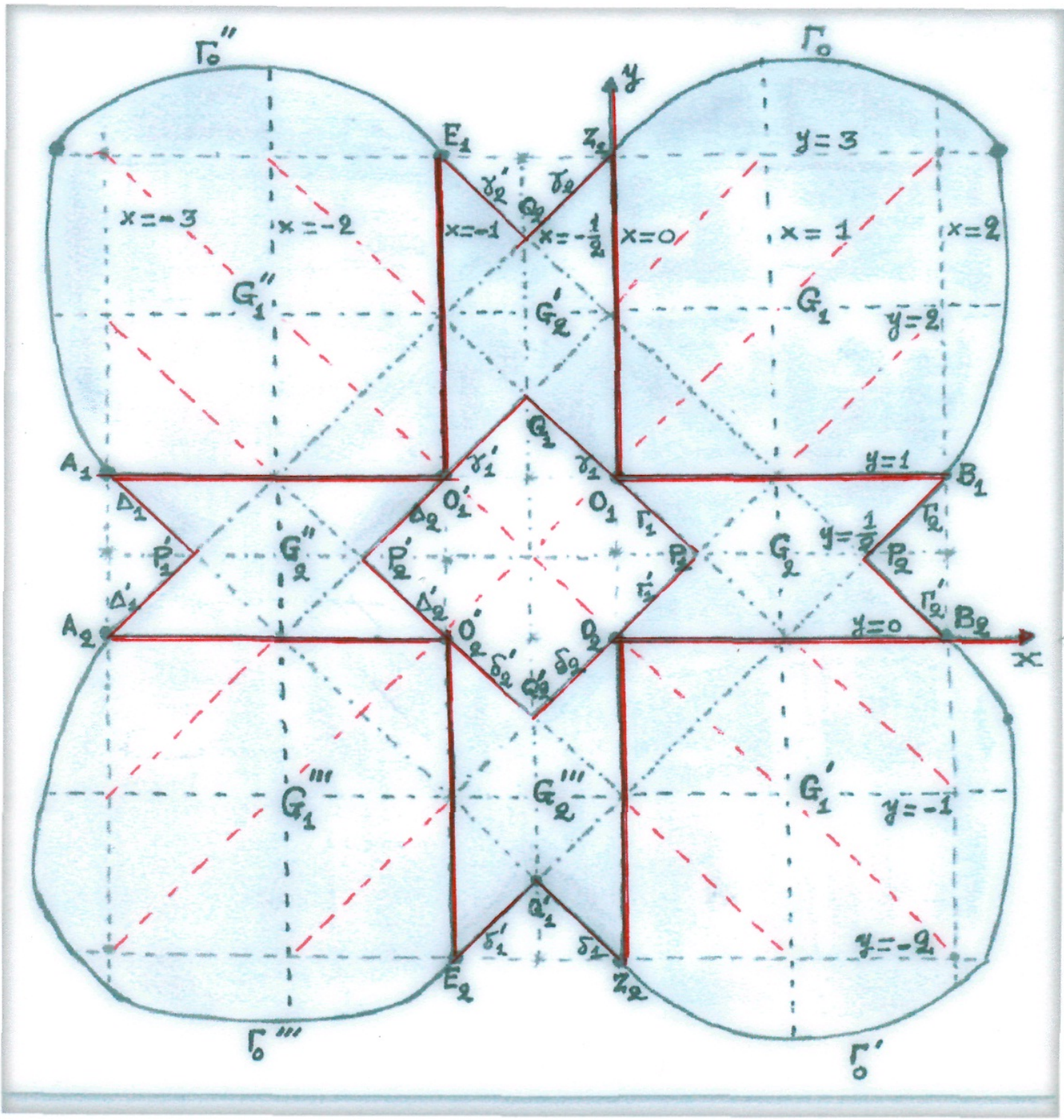

Figure 1. (Figure for Bitsadze-Lavrentjev problem: Tricomi case) 
The boundary $\partial D=\operatorname{Ext}(D) \cup \operatorname{Int}(D)$ of the doubly connected domain $D$ is formed by the following two exterior and interior boundaries

$$
\begin{gathered}
\operatorname{Ext}(D)=\left(\Gamma_{0} \cup \Gamma_{0}^{\prime} \cup \Gamma_{0}^{\prime \prime} \cup \Gamma_{0}^{\prime \prime \prime}\right) \cup\left(\Gamma_{2} \cup \Gamma_{2}^{\prime}\right) \cup\left(\gamma_{2} \cup \gamma_{2}^{\prime}\right) \cup\left(\Delta_{1} \cup \Delta_{1}^{\prime}\right) \cup\left(\delta_{1} \cup \delta_{1}^{\prime}\right), \\
\operatorname{Int}(D)=\left(\Gamma_{1} \cup \Gamma_{1}^{\prime}\right) \cup\left(\gamma_{1} \cup \gamma_{1}^{\prime}\right) \cup\left(\Delta_{2} \cup \Delta_{2}^{\prime}\right) \cup\left(\delta_{2} \cup \delta_{2}^{\prime}\right):
\end{gathered}
$$

the interior boundary of $D$, which is the boundary of the square:

$$
P_{1} Q_{1} P_{2}^{\prime} Q_{2}^{\prime}=P_{1} O_{1} Q_{1} O_{1}^{\prime} P_{2}^{\prime} O_{2}^{\prime} Q_{2}^{\prime} O_{2} P_{1}
$$

respectively: In the right hyperbolic domain $G_{2}=\{(x, y) \in D: 0<x<2,0<y<1\}$ with boundary $\partial G_{2}=\left(O_{1} B_{1}\right) \cup\left(O_{2} B_{2}\right) \cup\left(\Gamma_{1} \cup \Gamma_{1}^{\prime}\right) \cup\left(\Gamma_{2} \cup \Gamma_{2}^{\prime}\right)$, where $O_{1} B_{1}, O_{2} B_{2}$ are two parabolic lines with end points $O_{1}=(0,1), B_{1}=(2,1)$ and $O_{2}=(0,0), B_{2}=(2,0)$ and $\Gamma_{1}, \Gamma_{1}^{\prime}, \Gamma_{2}, \Gamma_{2}^{\prime}$ are four characteristics with end points: $O_{1}, B_{1}, O_{2}, B_{2} ; P_{1}=\left(\frac{1}{2}, \frac{1}{2}\right), P_{2}=\left(\frac{3}{2}, \frac{1}{2}\right)$, so that:

$$
\begin{gathered}
\Gamma_{1}=\left(O_{1} P_{1}\right): \int_{0}^{x} \sqrt{M(t)} d t=-\int_{1}^{y} \sqrt{-K(t)} d t \text { or } y=-x+1, \\
\Gamma_{1}^{\prime}=\left(P_{1} O_{2}\right): \int_{0}^{x} \sqrt{M(t)} d t=\int_{0}^{y} \sqrt{-K(t)} d t \text { or } y=x, \\
\Gamma_{2}=\left(P_{2} B_{1}\right): \int_{2}^{x} \sqrt{M(t)} d t=\int_{1}^{y} \sqrt{-K(t)} d t \text { or } y=x-1, \\
\Gamma_{2}^{\prime}=\left(B_{2} P_{2}\right): \int_{2}^{x} \sqrt{M(t)} d t=-\int_{0}^{y} \sqrt{-K(t)} d t \text { or } y=-x+2,
\end{gathered}
$$

where $K=-1 ; M=1$, such that on the characteristics: $H=-(d y)^{2}+(d x)^{2}=0$. In the upper hyperbolic domain $G_{2}^{\prime}=\{(x, y) \in D:-1<x<0,1<y<3\}$ with boundary $\partial G_{2}^{\prime}=\left(O_{1} Z_{1}\right) \cup$ $\left(O_{1}^{\prime} E_{1}\right) \cup\left(\gamma_{1} \cup \gamma_{1}^{\prime}\right) \cup\left(\gamma_{2} \cup \gamma_{2}^{\prime}\right)$, where $O_{1} Z_{1}, O_{1}^{\prime} E_{1}$ are two parabolic lines with end points $O_{1}=$ $(0,1), Z_{1}=(0,3)$ and $O_{1}^{\prime}=(-1,1), E_{1}=(-1,3)$ and $\gamma_{1}, \gamma_{1}^{\prime}, \gamma_{2}, \gamma_{2}^{\prime}$ are four characteristics with end points: $O_{1}, Z_{1}, O_{1}^{\prime}, E_{1} ; Q_{1}=\left(\frac{-1}{2}, \frac{3}{2}\right), Q_{2}=\left(\frac{-1}{2}, \frac{5}{2}\right)$, so that:

$$
\begin{gathered}
\gamma_{1}=\left(Q_{1} O_{1}\right): \int_{0}^{x} \sqrt{-M(t)} d t=-\int_{1}^{y} \sqrt{K(t)} d t \quad \text { or } y=-x+1, \quad \gamma_{1} \text { is the extension of } \Gamma_{1}, \\
\gamma_{1}^{\prime}=\left(O_{1}^{\prime} Q_{1}\right): \int_{-1}^{x} \sqrt{-M(t)} d t=\int_{1}^{y} \sqrt{K(t)} d t: \text { or } y=x+2, \\
\gamma_{2}=\left(Z_{1} Q_{2}\right): \int_{0}^{x} \sqrt{-M(t)} d t=\int_{3}^{y} \sqrt{K(t)} d t: \text { or } y=x+3,
\end{gathered}
$$




$$
\gamma_{2}^{\prime}=\left(Q_{2} E_{1}\right): \int_{-1}^{x} \sqrt{-M(t)} d t=-\int_{3}^{y} \sqrt{K(t)} d t \text { or } y=-x+2,
$$

where $K=1 ; M=-1$, such that on the characteristics: $-H=(d y)^{2}-(d x)^{2}=0$. In the left hyperbolic domain $G_{2}^{\prime \prime}=\{(x, y) \in D:-3<x<-1,0<y<1\}$ with boundary $\partial G_{2}^{\prime \prime}=\left(O_{1}^{\prime} A_{1}\right) \cup$ $\left(O_{2}^{\prime} A_{2}\right) \cup\left(\Delta_{1} \cup \Delta_{1}^{\prime}\right) \cup\left(\Delta_{2} \cup \Delta_{2}^{\prime}\right)$, where $O_{1}^{\prime} A_{1}, O_{2}^{\prime} A_{2}$ are two parabolic lines with end points $O_{1}^{\prime}=$ $(-1,1), A_{1}=(-3,1)$ and $O_{2}^{\prime}=(-1,0), A_{2}=(-3,0)$ and $\Delta_{1}, \Delta_{1}^{\prime}, \Delta_{2}, \Delta_{2}^{\prime}$ are four characteristics with end points: $O_{1}^{\prime}, A_{1}, O_{2}^{\prime}, A_{2} ; P_{1}^{\prime}=\left(\frac{-5}{2}, \frac{1}{2}\right), P_{2}^{\prime}=\left(\frac{-3}{2}, \frac{1}{2}\right)$, so that:

$$
\begin{gathered}
\Delta_{1}=\left(A_{1} P_{1}^{\prime}\right): \int_{-3}^{x} \sqrt{M(t)} d t=-\int_{1}^{y} \sqrt{-K(t)} d t \text { or } y=-x-2, \\
\Delta_{1}^{\prime}=\left(P_{1}^{\prime} A_{2}\right): \int_{-3}^{x} \sqrt{M(t)} d t=\int_{0}^{y} \sqrt{-K(t)} d t \text { or } y=x+3, \Delta_{1}^{\prime} \text { and } \gamma_{2} \text { lie on } A_{2} Z_{1}, \\
\Delta_{2}=\left(P_{2}^{\prime} O_{1}^{\prime}\right): \int_{-1}^{x} \sqrt{M(t)} d t=\int_{1}^{y} \sqrt{-K(t)} d t \text { or } y=x+2, \quad \Delta_{2} \text { is an extension of } \gamma_{1}^{\prime}, \\
\Delta_{2}^{\prime}=\left(O_{2}^{\prime} P_{2}^{\prime}\right): \int_{-1}^{x} \sqrt{M(t)} d t=-\int_{0}^{y} \sqrt{-K(t)} d t \text { or } y=-x-1,
\end{gathered}
$$

where $K=-1 ; M=1$, such that on the characteristics: $H=-(d y)^{2}+(d x)^{2}=0$. In the lower hyperbolic domain $G_{2}^{\prime \prime \prime}=\{(x, y) \in D:-1<x<0,-2<y<0\}$ with boundary $\partial G_{2}^{\prime \prime \prime}=\left(O_{2} Z_{2}\right) \cup$ $\left(O_{2}^{\prime} E_{2}\right) \cup\left(\delta_{1} \cup \delta_{1}^{\prime}\right) \cup\left(\delta_{2} \cup \delta_{2}^{\prime}\right)$, where $O_{2} Z_{2}, O_{2}^{\prime} E_{2}$ are two parabolic lines with end points $O_{2}=$ $(0,0), Z_{2}=(0,-2)$ and $O_{2}^{\prime}=(-1,0), E_{2}=(-1,-2)$ and $\delta_{1}, \delta_{1}^{\prime}, \delta_{2}, \delta_{2}^{\prime}$ are four characteristics with end points: $O_{2}, O_{2}^{\prime}, E_{2}, Z_{2} ; Q_{1}^{\prime}=\left(\frac{-1}{2}, \frac{-3}{2}\right), Q_{2}^{\prime}=\left(\frac{-1}{2}, \frac{-1}{2}\right)$, so that:

$$
\begin{gathered}
\delta_{1}=\left(Q_{1}^{\prime} Z_{2}\right): \int_{0}^{x} \sqrt{-M(t)} d t=-\int_{-2}^{y} \sqrt{K(t)} d t \text { or } y=-x-2, \delta_{1} \text { and } \Delta_{1} \text { lie on } A_{1} Z_{2}, \\
\delta_{1}^{\prime}=\left(E_{2} Q_{1}^{\prime}\right): \int_{-1}^{x} \sqrt{-M(t)} d t=\int_{-2}^{y} \sqrt{K(t)} d t \text { or } y=x-1, \delta_{1}^{\prime} \text { and } \Gamma_{2} \text { lie on } B_{1} E_{2}, \\
\delta_{2}=\left(O_{2} Q_{2}^{\prime}\right): \int_{0}^{x} \sqrt{-M(t)} d t=\int_{0}^{y} \sqrt{K(t)} d t \text { or } y=x, \quad \delta_{2} \text { is an extension of } \Gamma_{1}^{\prime}, \\
\delta_{2}^{\prime}=\left(Q_{2}^{\prime} O_{2}^{\prime}\right): \int_{-1}^{x} \sqrt{-M(t)} d t=-\int_{0}^{y} \sqrt{K(t)} d t \text { or } y=-x-1, \quad \delta_{2}^{\prime} \text { is an extension of } \Gamma_{1}^{\prime},
\end{gathered}
$$


where $K=1 ; M=-1$, such that on the characteristics: $-H=(d y)^{2}-(d x)^{2}=0$. Note that $K=M=-1$, in the interior of the square $P_{1} Q_{1} P_{2}^{\prime} Q_{2}^{\prime}$, which contains no part of the mixed domain $D$.

In the upper right elliptic domain $G_{1}=\{(x, y) \in D: x>0, y>1\}$ with boundary $\partial G_{1}=$ $\left(O_{1} B_{1}\right) \cup\left(O_{1} Z_{1}\right) \cup \Gamma_{0}$, where $O_{1} B_{1}, O_{1} Z_{1}$ are two parabolic lines with end points $O_{1}=(0,1), B_{1}=$ $(2,1)$ and $O_{1}=(0,1), Z_{1}=(0,3)$ and $\Gamma_{0}$ is the upper right elliptic arc connecting points $B_{1}=$ $(2,1), Z_{1}=(0,3)$.

In the lower right elliptic domain $G_{1}^{\prime}=\{(x, y) \in D: x>0, y<0\}$ with boundary $\partial G_{1}^{\prime}=\left(O_{2} B_{2}\right) \cup$ $\left(\mathrm{O}_{2} Z_{2}\right) \cup \Gamma_{0}^{\prime}$, where $\mathrm{O}_{2} B_{2}, \mathrm{O}_{2} Z_{2}$ are two parabolic lines with end points $O_{2}=(0,0), B_{2}=(2,0)$ and $\mathrm{O}_{2}=(0,0), Z_{2}=(0,-2)$ and $\Gamma_{0}^{\prime}$ is the lower right elliptic arc connecting points $B_{2}=(2,0), Z_{2}=$ $(0,-2)$.

In the upper left elliptic domain $G_{1}^{\prime \prime}=\{(x, y) \in D: x<-1, y>1\}$ with boundary $\partial G_{1}^{\prime \prime}=\left(O_{1}^{\prime} E_{1}\right) \cup$ $\left(O_{1}^{\prime} A_{1}\right) \cup \Gamma_{0}^{\prime \prime}$, where $O_{1}^{\prime} E_{1}, O_{1}^{\prime} A_{1}$ are two parabolic lines with end points $O_{1}^{\prime}=(-1,1), E_{1}=(-1,3)$ and $O_{1}^{\prime}=(-1,1), A_{1}=(-3,1)$ and $\Gamma_{0}^{\prime \prime}$ is the upper left elliptic arc connecting points $A_{1}=(-3,1)$ and $E_{1}=(-1,3)$.

In the lower left elliptic domain $G_{1}^{\prime \prime \prime}=\{(x, y) \in D: x<-1, y<0\}$ with boundary $\partial G_{1}^{\prime \prime \prime}=\left(O_{2}^{\prime} A_{2}\right) \cup$ $\left(O_{2}^{\prime} E_{2}\right) \cup \Gamma_{0}^{\prime \prime \prime}$, where $O_{2}^{\prime} E_{2}, O_{2}^{\prime} A_{2}$ are two parabolic lines with end points $O_{2}^{\prime}=(-1,0), E_{2}=(-1,-2)$ and $O_{2}^{\prime}=(-1,0), A_{2}=(-3,0)$ and $\Gamma_{0}^{\prime \prime \prime}$ is the lower left elliptic arc connecting points $A_{2}=(-3,0)$ and $E_{2}=(-1,-2)$. Let us consider the intersection points of the hyperbolic characteristics:

$\Gamma_{1} \cap \Gamma_{1}^{\prime}=\left\{P_{1}\right\}$, where $P_{1}=\left(\frac{1}{2}, \frac{1}{2}\right) ; \Gamma_{2} \cap \Gamma_{2}^{\prime}=\left\{P_{2}\right\}$, where $P_{2}=\left(\frac{3}{2}, \frac{1}{2}\right)$;

$\Delta_{1} \cap \Delta_{1}^{\prime}=\left\{P_{1}^{\prime}\right\}$, where $P_{1}^{\prime}=\left(\frac{-5}{2}, \frac{1}{2}\right) ; \Delta_{2} \cap \Delta_{2}^{\prime}=\left\{P_{2}^{\prime}\right\}$, where $P_{2}^{\prime}=\left(\frac{-3}{2}, \frac{1}{2}\right)$;

$\gamma_{1} \cap \gamma_{1}^{\prime}=\left\{Q_{1}\right\}$, where $Q_{1}=\left(\frac{-1}{2}, \frac{3}{2}\right) ; \gamma_{2} \cap \gamma_{2}^{\prime}=\left\{Q_{2}\right\}$, where $Q_{2}=\left(\frac{-1}{2}, \frac{5}{2}\right) ;$

$\delta_{1} \cap \delta_{1}^{\prime}=\left\{Q_{1}^{\prime}\right\}$, where $Q_{1}^{\prime}=\left(\frac{-1}{2}, \frac{-3}{2}\right) ; \delta_{2} \cap \delta_{2}^{\prime}=\left\{Q_{2}^{\prime}\right\}$, where $Q_{2}^{\prime}=\left(\frac{-1}{2}, \frac{-1}{2}\right)$.

Note that:

1. The boundary $\partial D$ is assumed to be a piecewise continuously differentiable arc. The elliptic arcs are "star-shaped" (counterclockwise).

2. We consider continuous solutions $u$ of the quaterelliptic -quaterhyperbolic equation $(*)$ with eight parabolic lines, which have the property that $u_{x}, u_{y}$ are continuous in the closure $\bar{D}=D \cup \partial D$.

These continuity conditions may be weakened at the eight "singular" points $A_{1}, A_{2}, B_{1}, B_{2}, O_{1}, O_{2}, O_{1}^{\prime}, O_{2}^{\prime}$, by considering $u_{x}, u_{y}$ continuous on the boundary $\partial D$ except at these points.By "quaterelliptic" and "quaterhyperbolic" we mean that Bitsadze-Lavrentjev equation (1.2) is elliptic in four different subdomains and hyperbolic in four other "symmetric" subdomains of the whole domain $D$.

In fact, equation (1.2) is elliptic and hyperbolic in $G_{1} \cup G_{1}^{\prime} \cup G_{1}^{\prime \prime} \cup G_{1}^{\prime \prime \prime}$ and $G_{2} \cup G_{2}^{\prime} \cup G_{2}^{\prime \prime} \cup G_{2}^{\prime \prime \prime}$, respectively.

Definition 2.1. A function $u=u(x, y)$ is a quasi-regular solution ([7], [8], [10]-[16]) of Problem (BL) if

i) $u \in C^{2}(D) \cap C(\bar{D}), \quad \bar{D}=D \cup \partial D$; 
ii) the Green's theorem (of the integral calculus) is applicable to the integrals

$$
\iint_{D} u_{x} L u d x d y, \iint_{D} u_{y} L u d x d y
$$

iii) the boundary and region integrals, which arise, exist; and

iv) $u$ satisfies the Bitsadze-Lavrentjev equation (1.2) in $D$ and the following boundary condition on the exterior boundary $\operatorname{Ext}(D)$ :

$$
u=\left\{\begin{array}{ccccccc}
\varphi_{1}(s) & \text { on } & \Gamma_{0} & ; & \varphi_{2}(s) & \text { on } & \Gamma_{0}^{\prime} \\
\varphi_{3}(s) & \text { on } & \Gamma_{0}^{\prime \prime} & ; & \varphi_{4}(s) & \text { on } & \Gamma_{0}^{\prime \prime \prime} \\
\psi_{1}(x) & \text { on } & \Gamma_{2} & ; & \psi_{2}(x) & \text { on } & \Gamma_{2}^{\prime} \\
\psi_{3}(x) & \text { on } & \gamma_{2} & ; & \psi_{4}(x) & \text { on } & \gamma_{2}^{\prime} \\
\psi_{5}(x) & \text { on } & \Delta_{1} & ; & \psi_{6}(x) & \text { on } & \Delta_{1}^{\prime} \\
\psi_{7}(x) & \text { on } & \delta_{1} & ; & \psi_{8}(x) & \text { on } & \delta_{1}^{\prime}
\end{array}\right.
$$

with continuous prescribed values.

\section{The exterior Bitsadze-Lavrentjev problem: Tricomi Case or Problem (BL-T):}

consists of finding a solution $u$ of the quaterelliptic -quaterhyperbolic equation (1.2) with eight parabolic lines in $D$ and which assumes continuous prescribed values (2.1).

Theorem 2.2. (UNIQUENESS THEOREM): Consider the general Bitsadze-Lavrentjev quaterelliptic - quaterhyperbolic equation (1.2) with eight parabolic lines and the boundary condition (2.1). Assume the above mixed doubly connected domain $D$ and the following conditions:

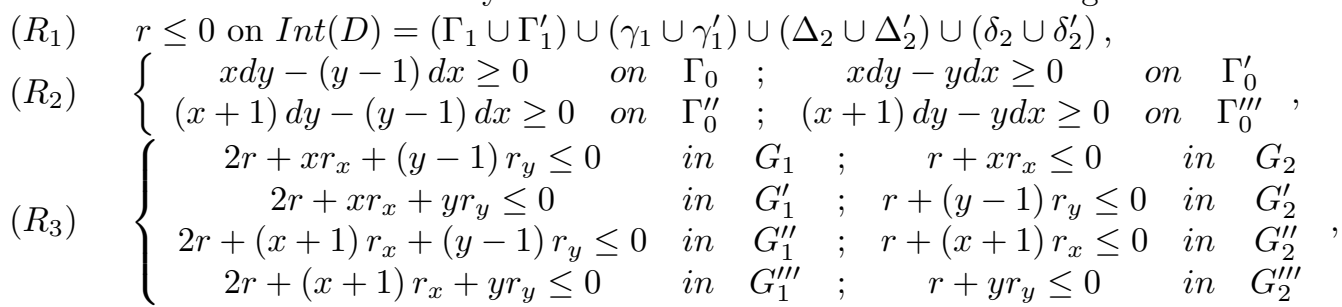

$\left(R_{4}\right) \quad K=M=+1$, in $G_{1} \cup G_{1}^{\prime} \cup G_{1}^{\prime \prime} \cup G_{1}^{\prime \prime \prime}$,

$\left(R_{5}\right) \quad\left\{\begin{array}{llll}K=-1 & , & M=+1 \quad \text { in } \quad G_{2} \cup G_{2}^{\prime \prime} \\ K=+1 & , & M=-1 & \text { in } \quad G_{2}^{\prime} \cup G_{2}^{\prime \prime \prime}\end{array}\right.$.

Let ()$_{x}=\partial() / \partial x,()^{\bullet}=d() / d x,()_{y}=\partial() / \partial y,()^{\prime}=d() / d y$,

where $f=f(x, y)$ is continuous in $D, r=r(x, y)$ is once-continuously differentiable in $D, K=$ $K(y)):=\operatorname{sgn}(y(y-1)|k(y)|)$ is a discontinuous function of $y$, for $y \in\left[-k_{1}, k_{2}\right]$, with $-k_{1}=\inf \{y$ : $(x, y) \in D\}$ and $k_{2}=\sup \{y:(x, y) \in D\}$, and $M=M(x):=\operatorname{sgn}(x(x+1)|m(x)|)$ is discontinuous for $x \in\left[-m_{1}, m_{2}\right]$ with $-m_{1}=\inf \{x:(x, y) \in D\}$ and $m_{2}=\sup \{x:(x, y) \in D\}$, such that $k=k(y) \neq 0 ; m=m(x) \neq 0$,everywhere in $D$, as well as $K=\left\{\begin{array}{ccc}+1 & \text { for } & \{y<0\} \cup\{y>1\} \\ 0 & \text { for } & \{y=0\} \cup\{y=1\} \\ -1 & \text { for } & \{0<y<1\}\end{array} \quad ; \quad M=\left\{\begin{array}{cccc}+1 & \text { for } & \{x<-1\} \cup\{x>0\} \\ 0 & \text { for } & \{x=0\} \cup\{x=-1\} . \\ -1 & \text { for } & \{-1<x<0\}\end{array}\right.\right.$.

Then Problem (BL-T) has at most one quasi-regular solution in $D$. 
Proof. We apply the well-known $a-b-c$ energy integral method with $a=0$, and use the above Bitsadze-Lavrentjev equation (1.2) and the boundary condition (2.1).

First, we assume two quasi-regular solutions $u_{1}, u_{2}$ of Problem (BL-T). Then we claim that $u=$ $u_{1}-u_{2}=0$ holds in the domain $D$. In fact, we investigate

$$
0=J=2\langle l u, L u\rangle_{0}=\iint_{D} 2 l u L u d x d y
$$

where $l u=b(x) u_{x}+c(y) u_{y}$, and $L u=L\left(u_{1}-u_{2}\right)=L u_{1}-L u_{2}=f-f=0$ in $D$, with choices

$$
b=b(x)=\left\{\begin{array}{ccc}
x & \text { in } & G_{1} \cup G_{1}^{\prime} \cup G_{2} \\
x+1 & \text { in } & G_{1}^{\prime \prime} \cup G_{1}^{\prime \prime} \cup G_{2}^{\prime \prime} \\
0 & \text { in } & G_{2}^{\prime} \cup G_{2}^{\prime \prime \prime}
\end{array}, \quad c=c(y)=\left\{\begin{array}{ccc}
y & \text { in } & G_{1}^{\prime} \cup G_{1}^{\prime \prime \prime} \cup G_{2}^{\prime \prime \prime} \\
y-1 & \text { in } & G_{1} \cup G_{1}^{\prime \prime} \cup G_{2}^{\prime} \\
0 & \text { in } & G_{2} \cup G_{2}^{\prime \prime}
\end{array} .\right.\right.
$$

We consider the new differential identities

$$
\begin{gathered}
2 b u_{x} u_{x x}=\left(b u_{x}^{2}\right)_{x}-(b)^{\bullet} u_{x}^{2} ; 2 b u_{x} u_{y y}=\left(2 b u_{x} u_{y}\right)_{y}-\left(b u_{y}^{2}\right)_{x}+(b)^{\bullet} u_{y}^{2}, \\
2 c u_{y} u_{x x}=\left(2 c u_{x} u_{y}\right)_{x}-\left(c u_{x}^{2}\right)_{y}+(c)^{\prime} u_{x}^{2} ; 2 c u_{y} u_{y y}=\left(c u_{y}^{2}\right)_{y}-(c)^{\prime} u_{y}^{2}, \\
2 b r u u_{x}=\left(b r u^{2}\right)_{x}-(b r)_{x} u^{2} ; 2 c r u u_{y}=\left(c r u^{2}\right)_{y}-(c r)_{y} u^{2} .
\end{gathered}
$$

Employing these identities and the classical Green's theorem of the integral calculus separately in $G_{i}^{j}(i=1,2 ; j=0,1,2,3)$, due to the discontinuity of $K, M$, we obtain from $(1.2),(2.2)-(2.3)$ that

$$
0=J_{G_{i}^{j}}=I_{G_{i}^{j}}+I_{\partial G_{i}^{j}}=\int_{G_{i}^{j}} 2\left[b u_{x}+c u_{y}\right]\left[K(y) u_{x x}+M(x) u_{y y}+r u\right] d x d y,
$$

where, if we denote:

$$
G_{1}=G_{1}^{0}, G_{1}^{\prime}=G_{1}^{1}, G_{1}^{\prime \prime}=G_{1}^{2}, G_{1}^{\prime \prime \prime}=G_{1}^{3} \quad ; \quad G_{2}=G_{2}^{0}, G_{2}^{\prime}=G_{2}^{1}, G_{2}^{\prime \prime}=G_{2}^{2}, G_{2}^{\prime \prime \prime}=G_{2}^{3},
$$

then

$$
\begin{aligned}
& I_{G_{i}^{j}}=\int_{G_{i}^{j}} Q\left(u_{x}, u_{y}\right) d x d y=\int_{G_{i}^{j}}\left(A u_{x}^{2}+B u_{y}^{2}+\Gamma u^{2}+2 \Delta u_{x} u_{y}\right) d x d y \\
& I_{\partial G_{i}^{j}}=\int_{\partial G_{i}^{j}} \tilde{Q}\left(u_{x}, u_{y}\right) d s=\int_{\partial G_{i}^{j}}\left(\tilde{A} u_{x}^{2}+\tilde{B} u_{y}^{2}+\tilde{\Gamma} u^{2}+2 \tilde{\Delta} u_{x} u_{y}\right) d s,
\end{aligned}
$$

with

$$
Q=Q\left(u_{x}, u_{y}\right)=A u_{x}^{2}+B u_{y}^{2}+\Gamma u^{2}+2 \Delta u_{x} u_{y}
$$

where

$$
A=\left[K\left(-\dot{b}+c^{\prime}\right)\right], B=M\left[\dot{b}-c^{\prime}\right], \Gamma=-\left[(b r)_{x}+(c r)_{y}\right], \Delta=0
$$


in $G_{i}^{j}$, and

$$
\tilde{Q}=\tilde{Q}\left(u_{x}, u_{y}\right)=\tilde{A} u_{x}^{2}+\tilde{B} u_{y}^{2}+\tilde{\Gamma} u^{2}+2 \tilde{\Delta} u_{x} u_{y}
$$

where

$$
\tilde{A}=\left(b v_{1}-c v_{2}\right) K, \tilde{B}=\left(-b v_{1}+c v_{2}\right) M, \tilde{\Gamma}=\left(b v_{1}+c v_{2}\right) r, \tilde{\Delta}=b M v_{2}+c K v_{1},
$$

on $\partial G_{i}^{j}$, where $v=\left(v_{1}, v_{2}\right)=(d y / d s,-d x / d s)$ is the outer unit normal vector on the boundary $\partial G_{i}^{j}$, of the domain $G_{i}^{j}$, with $0<d s=\sqrt{(d x)^{2}+(d y)^{2}},|\nu|=1$, and

$$
\iint_{G_{i}^{j}}()_{x} d x d y=\int_{\partial G_{i}^{j}}() v_{1} d s, \iint_{G_{i}^{j}}()_{y} d x d y=\int_{\partial G_{i}^{j}}() v_{2} d s
$$

are the Green's integral formulas.

From the above conditions of the uniqueness theorem 2.2 , we obtain

$$
A=B=\left\{\begin{array}{lll}
1 & \text { in } & G_{2} \cup G_{2}^{\prime} \cup G_{2}^{\prime \prime} \cup G_{2}^{\prime \prime \prime} \\
0 & \text { in } & G_{1} \cup G_{1}^{\prime} \cup G_{1}^{\prime \prime} \cup G_{1}^{\prime \prime \prime}
\end{array} .\right.
$$

Also

$$
0 \leq \Gamma=\left\{\begin{array}{ccc}
-\left(2 r+x r_{x}+(y-1) r_{y}\right) & \text { in } & G_{1} \\
-\left(2 r+x r_{x}+y r_{y}\right) & \text { in } & G_{1}^{\prime} \\
-\left(2 r+(x+1) r_{x}+(y-1) r_{y}\right) & \text { in } & G_{1}^{\prime \prime} \\
-\left(2 r+(x+1) r_{x}+y r_{y}\right) & \text { in } & G_{1}^{\prime \prime \prime}
\end{array} ;=\left\{\begin{array}{ccc}
-\left(r+x r_{x}\right) & \text { in } & G_{2} \\
-\left(r+(y-1) r_{y}\right) & \text { in } & G_{2}^{\prime} \\
-\left(r+(x+1) r_{x}\right) & \text { in } & G_{2}^{\prime \prime} \\
-\left(r+y r_{y}\right) & \text { in } & G_{2}^{\prime \prime \prime}
\end{array}\right.\right.
$$

We note:

1. In the upper right elliptic region $\bar{G}_{1}=G_{1} \cup \partial G_{1}: 0=J_{G_{1}}=I_{G_{1}}+I_{\partial G_{1}}$ :

$$
\begin{aligned}
I_{G_{1}} & =-\iint_{G_{1}}\left(2 r+x r_{x}+(y-1) r_{y}\right) u^{2} d x d y \geq 0 ; \\
I_{\partial G_{1}} & =\int_{\Gamma_{0}}\left(x \nu_{1}+(y-1) \nu_{2}\right)\left(\nu_{1}^{2}+\nu_{2}^{2}\right) N^{2} d s \\
& +2 \int_{Z_{1} O_{1}}(y-1) \nu_{1} u_{x} u_{y} d s+2 \int_{O_{1} B_{1}} x \nu_{2} u_{x} u_{y} d s ;
\end{aligned}
$$

$\left.u\right|_{\Gamma_{0}}=0$ yields $u_{x}=N \nu_{1} ; u_{y}=N \nu_{2}, N=$ normalizing factor , implying:

$$
\begin{aligned}
& Q_{0}=Q_{0}\left(u_{x}, u_{y}\right):=\left(x \nu_{1}-(y-1) \nu_{2}\right) u_{x}^{2}+\left(-x \nu_{1}+(y-1) \nu_{2}\right) u_{y}^{2} \\
& +2\left(x \nu_{2}+(y-1) \nu_{1}\right) u_{x} u_{y}=\left(x \nu_{1}+(y-1) \nu_{2}\right)\left(\nu_{1}^{2}+\nu_{2}^{2}\right) N^{2}>0
\end{aligned} .
$$

Note that $K=M=1$ and $b=x, c=y-1$ in $G_{1}$, as well as $u=0$ on $\Gamma_{0}$. 
2. In the right hyperbolic region $\bar{G}_{2}=G_{2} \cup \partial G_{2}: 0=J_{G_{2}}=I_{G_{2}}+I_{\partial G_{2}}$ :

$$
\begin{gathered}
I_{G_{2}}=\iint_{G_{2}}\left[u_{x}^{2}+u_{y}^{2}-\left(r+x r_{x}\right) u^{2}\right] d x d y \geq 0 ; \\
I_{\partial G_{2}}=\int_{\Gamma_{1}} x\left[\left(u_{x}-u_{y}\right)^{2}-r u^{2}\right] d x+\int_{\Gamma_{1}^{\prime}}(-x)\left[\left(u_{x}+u_{y}\right)^{2}-r u^{2}\right] d x \\
+2 \int_{B_{1} O_{1}} x \nu_{2} u_{x} u_{y} d s+2 \int_{O_{2} B_{2}} x \nu_{2} u_{x} u_{y} d s ;
\end{gathered}
$$

as

$$
\begin{aligned}
I_{\Gamma_{1}} & =\int_{\Gamma_{1}} x\left[-\nu_{1}\left(u_{x}^{2}+u_{y}^{2}\right)+2 \nu_{2} u_{x} u_{y}+\nu_{1} r u^{2}\right] d s \\
& =-\int_{\Gamma_{1}} x\left[\left(u_{x}^{2}+u_{y}^{2}\right) d y+2 u_{x} u_{y} d x-r u^{2} d y\right] \\
& =\int_{\Gamma_{1}} x\left[\left(u_{x}-u_{y}\right)^{2}-r u^{2}\right] d x \geq 0
\end{aligned}
$$

where on $\Gamma_{1}: y=-x+1$, or $d y=-d x: \nu_{1}=\nu_{2}$, and $\left.x d x\right|_{\Gamma_{1}}=-\left.x \nu_{2} d s\right|_{\Gamma_{1}}>0$, as well as

$$
\begin{aligned}
I_{\Gamma_{1}^{\prime}} & =\int_{\Gamma_{1}^{\prime}} x\left[-\nu_{1}\left(u_{x}^{2}+u_{y}^{2}\right)+2 \nu_{2} u_{x} u_{y}+\nu_{1} r u^{2}\right] d s \\
& =-\int_{\Gamma_{1}^{\prime}} x\left[\left(u_{x}^{2}+u_{y}^{2}\right) d y+2 u_{x} u_{y} d x-r u^{2} d y\right] \\
& =\int_{\Gamma_{1}^{\prime}}(-x)\left[\left(u_{x}+u_{y}\right)^{2}-r u^{2}\right] d x \geq 0
\end{aligned}
$$

where on $\Gamma_{1}^{\prime}: y=x$, or $d y=d x: \nu_{1}=-\nu_{2}$, and $\left.(-x) d x\right|_{\Gamma_{1}^{\prime}}=\left.x \nu_{2} d s\right|_{\Gamma_{1}^{\prime}}>0$, as well as

$$
I_{\Gamma_{2}}=\int_{\Gamma_{2}}(-x)\left[\left(u_{x}+u_{y}\right)^{2}-r u^{2}\right] d x=0 ;
$$

and

$$
I_{\Gamma_{2}^{\prime}}=\int_{\Gamma_{2}^{\prime}} x\left[\left(u_{x}-u_{y}\right)^{2}-r u^{2}\right] d x=0:
$$

$\left.u\right|_{\Gamma_{2} \cup \Gamma_{2}^{\prime}}=0$ yields $u_{x}=N \nu_{1} ; u_{y}=N \nu_{2}, N=$ normalizing factor, implying: $\left.\left(u_{x}+u_{y}\right)\right|_{\Gamma_{2}}=$ $\left.\left[\left(\nu_{1}+\nu_{2}\right) N\right]\right|_{\Gamma_{2}}=\left.\left[\left(\frac{d y-d x}{d s}\right) N\right]\right|_{\Gamma_{2}}=0$, as on $\quad \Gamma_{2}: y=x-1$, or $d y=d x$, and 
$\left.\left(u_{x}-u_{y}\right)\right|_{\Gamma_{2}^{\prime}}=\left.\left[\left(\nu_{1}-\nu_{2}\right) N\right]\right|_{\Gamma_{2}^{\prime}}=\left.\left[\left(\frac{d y+d x}{d s}\right) N\right]\right|_{\Gamma_{2}^{\prime}}=0$, as on $\Gamma_{2}^{\prime}: y=-x+2$, or $d y=$ $-d x ; I_{O_{1} B_{1}}+I_{B_{1} O_{1}}=0$, because $\left.\nu_{2}\right|_{O_{1} B_{1}}=-\left.\nu_{2}\right|_{B_{1} O_{1}}$.

Note that $K=-1, M=1$ and $b=x, c=0$ in $G_{2}$, as well as $u=0$ on $\Gamma_{2} \cup \Gamma_{2}^{\prime}$.

3. In the lower right elliptic region $\bar{G}_{1}^{\prime}=G_{1}^{\prime} \cup \partial G_{1}^{\prime}: 0=J_{G_{1}^{\prime}}=I_{G_{1}^{\prime}}+I_{\partial G_{1}^{\prime}}$ :

$$
\begin{gathered}
I_{G_{1}^{\prime}}=-\iint_{G_{1}^{\prime}}\left(2 r+x r_{x}+y r_{y}\right) u^{2} d x d y \geq 0 \\
I_{\partial G_{1}^{\prime}}=\int_{\Gamma_{0}^{\prime}}\left(x \nu_{1}+y \nu_{2}\right)\left(\nu_{1}^{2}+\nu_{2}^{2}\right) N^{2} d s+2 \int_{B_{2} O_{2}} x \nu_{2} u_{x} u_{y} d s+2 \int_{O_{2} Z_{2}} y \nu_{1} u_{x} u_{y} d s ;
\end{gathered}
$$

$\left.u\right|_{\Gamma_{0}^{\prime}}=0$ yields $u_{x}=N \nu_{1} ; u_{y}=N \nu_{2}, N=$ normalizing factor, implying:

$$
\begin{aligned}
Q_{1} & =Q_{1}\left(u_{x}, u_{y}\right): \\
& =\left(x \nu_{1}-y \nu_{2}\right)\left(u_{x}^{2}-u_{y}^{2}\right)+2\left(x \nu_{2}+y \nu_{1}\right) u_{x} u_{y} \\
& =\left(x \nu_{1}+y \nu_{2}\right)\left(\nu_{1}^{2}+\nu_{2}^{2}\right) N^{2}>0
\end{aligned}
$$

$I_{O_{2} B_{2}}+I_{B_{2} O_{2}}=0$, because $\left.\nu_{2}\right|_{O_{2} B_{2}}=-\left.\nu_{2}\right|_{B_{2} O_{2}}$. Note that $K=M=1$ and $b=x, c=y$ in $G_{1}$, as well as $u=0$ on $\Gamma_{0}^{\prime}$.

4. In the upper hyperbolic region $\bar{G}_{2}^{\prime}=G_{2}^{\prime} \cup \partial G_{2}^{\prime}: 0=J_{G_{2}^{\prime}}=I_{G_{2}^{\prime}}+I_{\partial G_{2}^{\prime}}$ :

$$
\begin{aligned}
I_{G_{2}^{\prime}} & =\iint_{G_{2}^{\prime}}\left[u_{x}^{2}+u_{y}^{2}-\left(r+(y-1) r_{y}\right) u^{2}\right] d x d y \geq 0 \\
I_{\partial G_{2}^{\prime}} & =\int_{\gamma_{1}}(y-1)\left[\left(u_{x}-u_{y}\right)^{2}-r u^{2}\right] d x+\int_{\gamma_{1}^{\prime}}(y-1)\left[\left(u_{x}+u_{y}\right)^{2}-r u^{2}\right] d x \\
& +2 \int_{O_{1} Z_{1}}(y-1) \nu_{1} u_{x} u_{y} d s+2 \int_{E_{1} O_{1}^{\prime}}(y-1) \nu_{1} u_{x} u_{y} d s
\end{aligned}
$$

as

$$
\begin{aligned}
I_{\gamma_{1}} & =\int_{\gamma_{1}}(y-1)\left[-\nu_{2}\left(u_{x}^{2}+u_{y}^{2}\right)+2 \nu_{1} u_{x} u_{y}+\nu_{2} r u^{2}\right] d s \\
& =\int_{\gamma_{1}}(y-1)\left[\left(u_{x}^{2}+u_{y}^{2}\right) d x+2 u_{x} u_{y} d y-r u^{2} d x\right] \\
& =\int_{\gamma_{1}}(y-1)\left[\left(u_{x}-u_{y}\right)^{2}-r u^{2}\right] d x \geq 0,
\end{aligned}
$$


where on $\gamma_{1}\left(:\right.$ extension of $\left.\Gamma_{1}\right): y=-x+1$, or $d y=-d x: \nu_{1}=\nu_{2}$, and $\left.(y-1) d x\right|_{\gamma_{1}}=$ $-\left.(y-1) \nu_{2} d s\right|_{\gamma_{1}}>0$, as well as

$$
\begin{aligned}
I_{\gamma_{1}^{\prime}} & =\int_{\gamma_{1}^{\prime}}(y-1)\left[-\nu_{2}\left(u_{x}^{2}+u_{y}^{2}\right)+2 \nu_{1} u_{x} u_{y}+\nu_{2} r u^{2}\right] d s \\
& =\int_{\gamma_{1}^{\prime}}(y-1)\left[\left(u_{x}^{2}+u_{y}^{2}\right) d x+2 u_{x} u_{y} d y-r u^{2} d x\right] \\
& =\int_{\gamma_{1}^{\prime}}(y-1)\left[\left(u_{x}+u_{y}\right)^{2}-r u^{2}\right] d x \geq 0,
\end{aligned}
$$

where on $\gamma_{1}^{\prime}: y=x+2$, or $d y=d x: \nu_{1}=-\nu_{2}$, and $\left.(y-1) d x\right|_{\gamma_{1}^{\prime}}=-\left.(y-1) \nu_{2} d s\right|_{\gamma_{1}^{\prime}}>0$, as well as

$$
I_{\gamma_{2}}=\int_{\gamma_{2}}(y-1)\left[\left(u_{x}+u_{y}\right)^{2}-r u^{2}\right] d x=0
$$

and

$$
I_{\gamma_{2}^{\prime}}=\int_{\gamma_{2}^{\prime}}(y-1)\left[\left(u_{x}-u_{y}\right)^{2}-r u^{2}\right] d x=0:
$$

$\left.u\right|_{\gamma_{2} \cup \gamma_{2}^{\prime}}=0$ yields $u_{x}=N \nu_{1} ; u_{y}=N \nu_{2}, N=$ normalizing factor implying: $\left.\left(u_{x}+u_{y}\right)\right|_{\gamma_{2}}=$ $\left.\left[\left(\nu_{1}+\nu_{2}\right) N\right]\right|_{\gamma_{2}}=\left.\left[\left(\frac{d y-d x}{d s}\right) N\right]\right|_{\gamma_{2}}=0$, as on $\gamma_{2}: y=x+3$, or $d y=d x$, and $\left.\left(u_{x}-u_{y}\right)\right|_{\gamma_{2}^{\prime}}=$ $\left.\left[\left(\nu_{1}-\nu_{2}\right) N\right]\right|_{\gamma_{2}^{\prime}}=\left.\left[\left(\frac{d y+d x}{d s}\right) N\right]\right|_{\gamma_{2}^{\prime}}=0$, as on $\gamma_{2}^{\prime}: y=-x+2$, or $d y=-d x ; I_{Z_{1} O_{1}}+I_{O_{1} Z_{1}}=$ 0 , because $\left.\nu_{2}\right|_{Z_{1} O_{1}}=-\left.\nu_{2}\right|_{O_{1} Z_{1}}$. Note that $K=1, \quad M=-1$ and $b=0, c=y-1$ in $G_{2}^{\prime}$, as well as $u=0$ on $\gamma_{2} \cup \gamma_{2}^{\prime}$.

5. In the upper left elliptic region $\bar{G}_{1}^{\prime \prime}=G_{1}^{\prime \prime} \cup \partial G_{1}^{\prime \prime}: 0=J_{G_{1}^{\prime \prime}}=I_{G_{1}^{\prime \prime}}+I_{\partial G_{1}^{\prime \prime}}$ :

$$
\begin{aligned}
I_{G_{1}^{\prime \prime}} & =-\iint_{G_{1}^{\prime \prime}}\left(2 r+(x+1) r_{x}+(y-1) r_{y}\right) u^{2} d x d y \geq 0 ; \\
I_{\partial G_{1}^{\prime \prime}} & =\int_{\Gamma_{0}^{\prime \prime}}\left((x+1) \nu_{1}+(y-1) \nu_{2}\right)\left(\nu_{1}^{2}+\nu_{2}^{2}\right) N^{2} d s \\
& +2 \int_{A_{1} O_{1}^{\prime}}(x+1) \nu_{2} u_{x} u_{y} d s+2 \int_{O_{1}^{\prime} E_{1}}(y-1) \nu_{1} u_{x} u_{y} d s ;
\end{aligned}
$$

$\left.u\right|_{\Gamma_{0}^{\prime \prime}}=0$ yields relations

$$
u_{x}=N \nu_{1} ; u_{y}=N \nu_{2}
$$

$N=$ normalizing factor, implying:

$$
\begin{aligned}
Q_{2}= & Q_{2}\left(u_{x}, u_{y}\right):=\left((x+1) \nu_{1}-(y-1) \nu_{2}\right)\left(u_{x}^{2}-u_{y}^{2}\right) \\
& +2\left((x+1) \nu_{2}+(y-1) \nu_{1}\right) u_{x} u_{y} \\
= & \left((x+1) \nu_{1}+(y-1) \nu_{2}\right)\left(\nu_{1}^{2}+\nu_{2}^{2}\right) N^{2}>0
\end{aligned}
$$


$I_{E_{1} O_{1}^{\prime}}+I_{O_{1}^{\prime} E_{1}}=0$, because $\left.\nu_{1}\right|_{E_{1} O_{1}^{\prime}}=-\left.\nu_{1}\right|_{O_{1}^{\prime} E_{1}} \cdot I_{O_{2} B_{2}}+I_{B_{2} O_{2}}=0$, because $\left.\nu_{2}\right|_{O_{2} B_{2}}=$ $-\left.\nu_{2}\right|_{B_{2} O_{2}}$. Note that $K=M=1$ and $b=x+1, c=y-1$ in $G_{1}^{\prime \prime}$, as well as $u=0$ on $\Gamma_{0}^{\prime \prime}$.

6. In the left hyperbolic region: $\bar{G}_{2}^{\prime \prime}=G_{2}^{\prime \prime} \cup \partial G_{2}^{\prime \prime}: 0=J_{G_{2}^{\prime \prime}}=I_{G_{2}^{\prime \prime}}+I_{\partial G_{2}^{\prime \prime}}$ :

$$
\begin{aligned}
I_{G_{2}^{\prime \prime}}= & \iint_{G_{2}^{\prime \prime}}\left[u_{x}^{2}+u_{y}^{2}-\left(r+(x+1) r_{x}\right) u^{2}\right] d x d y \geq 0 \\
I_{\partial G_{2}^{\prime \prime}}= & \int_{\Delta_{2}^{\prime}}(x+1)\left[\left(u_{x}-u_{y}\right)^{2}-r u^{2}\right] d x+\int_{\Delta_{2}}(-(x+1))\left[\left(u_{x}+u_{y}\right)^{2}-r u^{2}\right] d x \\
& +2 \int_{O_{1}^{\prime} A_{1}}(x+1) \nu_{2} u_{x} u_{y} d s+2 \int_{A_{2} O_{2}^{\prime}}(x+1) \nu_{2} u_{x} u_{y} d s
\end{aligned}
$$

as

$$
\begin{aligned}
I_{\Delta_{2}} & =\int_{\Gamma_{1}}(x+1)\left[-\nu_{1}\left(u_{x}^{2}+u_{y}^{2}\right)+2 \nu_{2} u_{x} u_{y}+\nu_{1} r u^{2}\right] d s \\
& =-\int_{\Delta_{2}}(x+1)\left[\left(u_{x}^{2}+u_{y}^{2}\right) d y+2 u_{x} u_{y} d x-r u^{2} d y\right] \\
& =-\int_{\Delta_{2}}(x+1)\left[\left(u_{x}+u_{y}\right)^{2}-r u^{2}\right] d x \geq 0,
\end{aligned}
$$

where on $\Delta_{2}: y=x+2$, or $d y=d x: \nu_{1}=-\nu_{2}$, and $-\left.(x+1) d x\right|_{\Delta_{2}}=\left.(x+1) \nu_{2} d s\right|_{\Delta_{2}}>0$, as well as

$$
\begin{aligned}
I_{\Delta_{2}^{\prime}} & =\int_{\Delta_{2}^{\prime}}(x+1)\left[-\nu_{1}\left(u_{x}^{2}+u_{y}^{2}\right)+2 \nu_{2} u_{x} u_{y}+\nu_{1} r u^{2}\right] d s \\
& =-\int_{\Delta_{2}^{\prime}}(x+1)\left[\left(u_{x}^{2}+u_{y}^{2}\right) d y+2 u_{x} u_{y} d x-r u^{2} d y\right] \\
& =\int_{\Delta_{2}^{\prime}}(x+1)\left[\left(u_{x}-u_{y}\right)^{2}-r u^{2}\right] d x \geq 0,
\end{aligned}
$$

where on $\Delta_{2}^{\prime}: y=-x-1$, or $d y=-d x: \nu_{1}=\nu_{2}$, and $\left.(x+1) d x\right|_{\Delta_{2}^{\prime}}=-\left.(x+1) \nu_{2} d s\right|_{\Delta_{2}^{\prime}}>$ 0 , as well as

$$
I_{\Delta_{1}^{\prime}}=\int_{\Delta_{1}^{\prime}}(-(x+1))\left[\left(u_{x}+u_{y}\right)^{2}-r u^{2}\right] d x=0
$$

and

$$
I_{\Delta_{1}}=\int_{\Delta_{1}}(x+1)\left[\left(u_{x}-u_{y}\right)^{2}-r u^{2}\right] d x=0:
$$


$\left.u\right|_{\Delta_{1} \cup \Delta_{1}^{\prime}}=0$ yields,$u_{x}=N \nu_{1} ; u_{y}=N \nu_{2}, N=$ normalizing factor implying: $\left.\left(u_{x}+u_{y}\right)\right|_{\Delta_{1}^{\prime}}=\left.\left[\left(\nu_{1}+\nu_{2}\right) N\right]\right|_{\Delta_{1}^{\prime}}=\left.\left[\left(\frac{d y-d x}{d s}\right) N\right]\right|_{\Delta_{1}^{\prime}}=0$, as on $\Delta_{1}^{\prime}: y=x+3$, or $d y=d x$, and $\left.\left(u_{x}-u_{y}\right)\right|_{\Delta_{1}}=\left.\left[\left(\nu_{1}-\nu_{2}\right) N\right]\right|_{\Delta_{1}}=\left.\left[\left(\frac{d y+d x}{d s}\right) N\right]\right|_{\Delta_{1}}=0$, as on $\Delta_{1}: y=-x-$ 2 , or $d y=-d x ; I_{A_{1} O_{1}^{\prime}}+I_{O_{1}^{\prime} A_{1}}=0$, because $\left.\nu_{2}\right|_{A_{1} O_{1}^{\prime}}=-\left.\nu_{2}\right|_{O_{1}^{\prime} A_{1}}$. Note that $K=-1, M=1$ and $b=x+1, c=0$ in $G_{2}^{\prime \prime}$, as well as $u=0$ on $\Delta_{1} \cup \Delta_{1}^{\prime}$.

7. In the lower elliptic region: $\bar{G}_{1}^{\prime \prime \prime}=G_{1}^{\prime \prime \prime} \cup \partial G_{1}^{\prime \prime \prime}: 0=J_{G_{1}^{\prime \prime \prime}}=I_{G_{1}^{\prime \prime \prime}}+I_{\partial G_{1}^{\prime \prime \prime}}$ :

$$
\begin{aligned}
I_{G_{1}^{\prime \prime \prime}}= & -\iint_{G_{1}^{\prime \prime \prime}}\left(2 r+(x+1) r_{x}+y r_{y}\right) u^{2} d x d y \geq 0 ; \\
I_{\partial G_{1}^{\prime \prime \prime}}= & \int_{\Gamma_{0}^{\prime \prime \prime}}\left((x+1) \nu_{1}+y \nu_{2}\right)\left(\nu_{1}^{2}+\nu_{2}^{2}\right) N^{2} d s \\
& +2 \int_{E_{2} O_{2}^{\prime}} y \nu_{1} u_{x} u_{y} d s+2 \int_{O_{2}^{\prime} A_{2}}(x+1) \nu_{2} u_{x} u_{y} d s
\end{aligned}
$$

$\left.u\right|_{\Gamma_{0}^{\prime \prime \prime}}=0$ yields $u_{x}=N \nu_{1} ; u_{y}=N \nu_{2}, N=$ normalizing factor, implying:

$$
\begin{aligned}
Q_{3} & =Q_{3}\left(u_{x}, u_{y}\right): \\
& =\left((x+1) \nu_{1}-y \nu_{2}\right)\left(u_{x}^{2}-u_{y}^{2}\right)+2\left((x+1) \nu_{2}+y \nu_{1}\right) u_{x} u_{y} \\
& =\left((x+1) \nu_{1}+y \nu_{2}\right)\left(\nu_{1}^{2}+\nu_{2}^{2}\right) N^{2}>0 ;
\end{aligned}
$$

$I_{A_{2} O_{2}^{\prime}}+I_{O_{2}^{\prime} A_{2}}=0$, because $\left.\nu_{2}\right|_{A_{2} O_{2}^{\prime}}=-\left.\nu_{2}\right|_{O_{2}^{\prime} A_{2}}$.

Note that $K=M=1$ and $b=x+1, c=y$ in $G_{1}^{\prime \prime \prime}$, as well as $u=0$ on $\Gamma_{0}^{\prime \prime \prime}$.

8. In the lower hyperbolic region: $\bar{G}_{2}^{\prime \prime \prime}=G_{2}^{\prime \prime \prime} \cup \partial G_{2}^{\prime \prime \prime}: 0=J_{G_{2}^{\prime \prime \prime}}=I_{G_{2}^{\prime \prime \prime}}+I_{\partial G_{2}^{\prime \prime \prime}}$ :

$$
\begin{aligned}
I_{G_{2}^{\prime \prime \prime}}= & \iint_{G_{2}^{\prime \prime \prime}}\left[u_{x}^{2}+u_{y}^{2}-\left(r+y r_{y}\right) u^{2}\right] d x d y \geq 0 \\
I_{\partial G_{2}^{\prime \prime \prime}}= & \int_{\delta_{2}^{\prime}} y\left[\left(u_{x}-u_{y}\right)^{2}-r u^{2}\right] d x+\int_{\delta_{2}} y\left[\left(u_{x}+u_{y}\right)^{2}-r u^{2}\right] d x \\
& +2 \int_{Z_{2} O_{2}} y \nu_{1} u_{x} u_{y} d s+2 \int_{O_{2}^{\prime} E_{2}} y \nu_{1} u_{x} u_{y} d s ;
\end{aligned}
$$

as

$$
\begin{aligned}
I_{\delta_{2}} & =\int_{\delta_{2}} y\left[-\nu_{2}\left(u_{x}^{2}+u_{y}^{2}\right)+2 \nu_{1} u_{x} u_{y}+\nu_{2} r u^{2}\right] d s \\
& =\int_{\delta_{2}} y\left[\left(u_{x}^{2}+u_{y}^{2}\right) d x+2 u_{x} u_{y} d y-r u^{2} d x\right]=\int_{\delta_{2}} y\left[\left(u_{x}+u_{y}\right)^{2}-r u^{2}\right] d x \geq 0,
\end{aligned}
$$


where on $\delta_{2}\left(:\right.$ extension of $\left.\Gamma_{1}^{\prime}\right): y=x$, or $d y=d x: \nu_{1}=-\nu_{2}$, and $\left.y d x\right|_{\delta_{2}}=-\left.y \nu_{2} d s\right|_{\delta_{2}}>$ 0 , as well as

$$
\begin{aligned}
I_{\delta_{2}^{\prime}} & =\int_{\delta_{2}^{\prime}} y\left[-\nu_{2}\left(u_{x}^{2}+u_{y}^{2}\right)+2 \nu_{1} u_{x} u_{y}+\nu_{2} r u^{2}\right] d s=\int_{\delta_{2}^{\prime}} y\left[\left(u_{x}^{2}+u_{y}^{2}\right) d x+2 u_{x} u_{y} d y-r u^{2} d x\right] \\
& =\int_{\delta_{2}^{\prime}} y\left[\left(u_{x}-u_{y}\right)^{2}-r u^{2}\right] d x \geq 0,
\end{aligned}
$$

where on $\delta_{2}^{\prime}$ (extension of $\left.\Delta_{2}^{\prime}\right): y=-x-1$, or $d y=-d x \quad: \quad \nu_{1}=\nu_{2}$, and $\left.y d x\right|_{\delta_{2}^{\prime}}=$ $-\left.y \nu_{2} d s\right|_{\delta_{2}^{\prime}}>0$, as well as $I_{\delta_{1}}=\int_{\delta_{1}} y\left[\left(u_{x}-u_{y}\right)^{2}-r u^{2}\right] d x=0$;

and $I_{\delta_{1}^{\prime}}=\int_{\delta_{1}^{\prime}} y\left[\left(u_{x}+u_{y}\right)^{2}-r u^{2}\right] d x=0$ :

$\left.u\right|_{\delta_{1} \cup \delta_{1}^{\prime}}=0$ yields $u_{x}=N \nu_{1} ; u_{y}=N \nu_{2}, N=$ normalizing factor, implying: $\left.\left(u_{x}+u_{y}\right)\right|_{\delta_{1}^{\prime}}=$ $\left.\left[\left(\nu_{1}+\nu_{2}\right) N\right]\right|_{\delta_{1}^{\prime}}=\left.\left[\left(\frac{d y-d x}{d s}\right) N\right]\right|_{\delta_{1}^{\prime}}=0$, as on $\delta_{1}^{\prime}: y=x-1$, or $d y=d x$, and $\left.\left(u_{x}-u_{y}\right)\right|_{\delta_{1}}=$ $\left.\left[\left(\nu_{1}-\nu_{2}\right) N\right]\right|_{\delta_{1}}=\left.\left[\left(\frac{d y+d x}{d s}\right) N\right]\right|_{\delta_{1}}=0$, as on $\delta_{1}: y=-x-2$, or $d y=-d x ; I_{Z_{2} O_{2}}+I_{O_{2} Z_{2}}=$ 0 , because $\left.\nu_{1}\right|_{Z_{2} O_{2}}=-\left.\nu_{1}\right|_{O_{2} Z_{2}} \cdot I_{E_{2} O_{2}^{\prime}}+I_{O_{2}^{\prime} E_{2}}=0$, because $\left.\nu_{1}\right|_{E_{2} O_{2}^{\prime}}=-\left.\nu_{1}\right|_{O_{2}^{\prime} E_{2}}$. Note : $K=1, \quad M=-1$ and $b=0, c=y$ in $G_{2}^{\prime \prime \prime}$, and $u=0$ on $\delta_{1} \cup \delta_{1}^{\prime}$. On the eight parabolic segments, the following "vanishing" relation holds:

$$
\begin{array}{r}
\left(I_{O_{1} B_{1}}+I_{B_{1} O_{1}}\right)+\left(I_{O_{2} B_{2}}+I_{B_{2} O_{2}}\right)+\left(I_{O_{1} Z_{1}}+I_{Z_{1} O_{1}}\right) \\
+\left(I_{O_{2} Z_{2}}+I_{Z_{2} O_{2}}\right)+\left(I_{O_{1}^{\prime} A_{1}}+I_{A_{1} O_{1}^{\prime}}\right)+\left(I_{O_{2}^{\prime} A_{2}}+I_{A_{2} O_{2}^{\prime}}\right) \\
+\left(I_{O_{1}^{\prime} E_{1}}+I_{E_{1} O_{1}^{\prime}}\right)+\left(I_{O_{1}^{\prime} E_{2}}+I_{E_{2} O_{2}^{\prime}}\right)=0 .
\end{array}
$$

Also on the four pairs of characteristics: $\Gamma_{2}, \Gamma_{2}^{\prime} ; \gamma_{2}, \gamma_{2}^{\prime} ; \Delta_{1}, \Delta_{1}^{\prime} ; \delta_{1}, \delta_{1}^{\prime}$ :

$$
\left(I_{\Gamma_{2}}+I_{\Gamma_{2}^{\prime}}\right)+\left(I_{\gamma_{2}}+I_{\gamma_{2}^{\prime}}\right)+\left(I_{\Delta_{1}}+I_{\Delta_{1}^{\prime}}\right)+\left(I_{\delta_{1}}+I_{\delta_{1}^{\prime}}\right)=0
$$

Applying the energy integral method in each of the eight regions: $G_{i}, G_{i}^{\prime}, G_{i}^{\prime \prime}, G_{i}^{\prime \prime \prime}, \quad i=1,2$, separately, due to the discontinuity of

$$
K=\left\{\begin{array}{ccc}
+1 & \text { for } & \{y<0\} \cup\{y>1\} \\
0 & \text { for } & \{y=0\} \cup\{y=1\} \\
-1 & \text { for } & \{0<y<1\}
\end{array} \quad ; M=\left\{\begin{array}{ccc}
+1 & \text { for } & \{x<-1\} \cup\{x>0\} \\
0 & \text { for } & \{x=0\} \cup\{x=-1\} \\
-1 & \text { for } & \{-1<x<0\}
\end{array},\right.\right.
$$

in $D$, Green's theorem cannot be applied directly in the whole mixed domain $D$, and then adding all the pertinent integral expressions, we find $0=J_{D}=I_{D}+I_{\partial D} \geq 0$, where $I_{D} \geq$ 
$0 ; I_{\partial D} \geq 0$. In fact,

$$
\begin{aligned}
I_{D}= & -\iint_{G_{1}}\left(2 r+x r_{x}+(y-1) r_{y}\right) u^{2} d x d y-\iint_{G_{1}^{\prime}}\left(2 r+x r_{x}+y r_{y}\right) u^{2} d x d y \\
& -\iint_{G_{1}^{\prime \prime}}\left(2 r+(x+1) r_{x}+(y-1) r_{y}\right) u^{2} d x d y \\
& -\iint_{G_{1}^{\prime \prime \prime}}\left(2 r+(x+1) r_{x}+y r_{y}\right) u^{2} d x d y+\iint_{G_{2} \cup G_{2}^{\prime} \cup G_{2}^{\prime \prime} \cup G_{2}^{\prime \prime \prime}}\left(u_{x}^{2}+u_{y}^{2}\right) d x d y \\
& -\iint_{G_{2}}\left(r+x r_{x}\right) u^{2} d x d y-\iint_{G_{2}^{\prime}}\left(r+(y-1) r_{y}\right) u^{2} d x d y \\
& -\iint_{G_{2}^{\prime \prime}}\left(r+(x+1) r_{x}\right) u^{2} d x d y-\iint_{G_{2}^{\prime \prime}}\left(r+y r_{y}\right) u^{2} d x d y \geq 0 .
\end{aligned}
$$

Also

$$
\begin{aligned}
I_{\partial D}= & \int_{\Gamma_{0}}\left(x \nu_{1}+(y-1) \nu_{2}\right)\left(\nu_{1}^{2}+\nu_{2}^{2}\right) N^{2} d s+\int_{\Gamma_{0}^{\prime}}\left(x \nu_{1}+y \nu_{2}\right)\left(\nu_{1}^{2}+\nu_{2}^{2}\right) N^{2} d s \\
& +\int_{\Gamma_{0}^{\prime \prime}}\left((x+1) \nu_{1}+(y-1) \nu_{2}\right)\left(\nu_{1}^{2}+\nu_{2}^{2}\right) N^{2} d s \\
& +\int_{\Gamma_{0}^{\prime \prime \prime}}\left((x+1) \nu_{1}+y \nu_{2}\right)\left(\nu_{1}^{2}+\nu_{2}^{2}\right) N^{2} d s+\int_{\Gamma_{1}} x\left[\left(u_{x}-u_{y}\right)^{2}-r u^{2}\right] d x \\
& -\int_{\Gamma_{1}^{\prime}} x\left[\left(u_{x}+u_{y}\right)^{2}-r u^{2}\right] d x+\int(y-1)\left[\left(u_{x}-u_{y}\right)^{2}-r u^{2}\right] d x \\
& +\int_{\gamma_{1}^{\prime}}(y-1)\left[\left(u_{x}+u_{y}\right)^{2}-r u^{2}\right] d x-\int_{\Delta_{2}}(x+1)\left[\left(u_{x}+u_{y}\right)^{2}-r u^{2}\right] d x \\
& +\int_{\Delta_{2}^{\prime}}(x+1)\left[\left(u_{x}-u_{y}\right)^{2}-r u^{2}\right] d x+\int_{\delta_{2}} y\left[\left(u_{x}+u_{y}\right)^{2}-r u^{2}\right] d x \\
& +\int_{\delta_{2}^{\prime}} y\left[\left(u_{x}-u_{y}\right)^{2}-r u^{2}\right] d x \geq 0 .
\end{aligned}
$$

From the maximum principle and the uniqueness of the solution of the Cauchy problem on hyperbolic equations, one obtains $u=u(x, y) \equiv 0$, everywhere in the whole mixed domain $D$, completing the proof of our uniqueness theorem. In fact, from the maximum principle, $\left.u\right|_{\bar{G}_{2} \cup \bar{G}_{2}^{\prime} \cup \bar{G}_{2}^{\prime \prime} \cup \bar{G}_{2}^{\prime \prime \prime}}=0$, implies that $\left.u\right|_{\bar{G}_{1} \cup \bar{G}_{1}^{\prime} \cup \bar{G}_{1}^{\prime \prime} \cup \bar{G}_{1}^{\prime \prime \prime}}=0$. 


\section{Exterior Bitsadze-Lavrentjev problem: Frankl Case}

Consider the general Bitsadze-Lavrentjev quaterelliptic - quaterhyperbolic equation (1.2) with eight parabolic lines of degeneracy in a bounded doubly connected mixed domain $\tilde{D}$ with a piecewise smooth boundary

$$
\partial \tilde{D}=\operatorname{Ext}(\tilde{D}) \cup \operatorname{Int}(\tilde{D})=[\operatorname{ExtEl}(\tilde{D}) \cup \operatorname{ExtHn}(\tilde{D})] \cup \operatorname{Int}(\tilde{D}),
$$

where $\tilde{D}$ is a part of $D$, and $\operatorname{Int}(\tilde{D})=\operatorname{Int}(D)$, as well as

$$
\operatorname{ExtEl}(\tilde{D})=\Gamma_{0} \cup \Gamma_{0}^{\prime} \cup \Gamma_{0}^{\prime \prime} \cup \Gamma_{0}^{\prime \prime \prime},
$$

is the elliptic exterior boundary of $\tilde{D}$ and

$$
\operatorname{ExtHn}(\tilde{D})=\left(\tilde{\Gamma}_{2} \cup \tilde{\Gamma}_{2}^{\prime}\right) \cup\left(\tilde{\gamma}_{2} \cup \tilde{\gamma}_{2}^{\prime}\right) \cup\left(\tilde{\Delta}_{1} \cup \tilde{\Delta}_{1}^{\prime}\right) \cup\left(\tilde{\delta}_{1} \cup \tilde{\delta}_{1}^{\prime}\right)
$$

is the non-characteristic hyperbolic exterior boundary of $\tilde{D}$, such that:

$$
\begin{aligned}
\operatorname{Ext}(\tilde{D})= & \operatorname{Ext} \operatorname{El}(\tilde{D}) \cup \operatorname{ExtHn}(\tilde{D})=\left(\Gamma_{0} \cup \Gamma_{0}^{\prime} \cup \Gamma_{0}^{\prime \prime} \cup \Gamma_{0}^{\prime \prime \prime}\right) \\
& \cup\left[\left(\tilde{\Gamma}_{2} \cup \tilde{\Gamma}_{2}^{\prime}\right) \cup\left(\tilde{\gamma}_{2} \cup \tilde{\gamma}_{2}^{\prime}\right) \cup\left(\tilde{\Delta}_{1} \cup \tilde{\Delta}_{1}^{\prime}\right) \cup\left(\tilde{\delta}_{1} \cup \tilde{\delta}_{1}^{\prime}\right)\right],
\end{aligned}
$$

is the exterior boundary of $\tilde{D}$, with the following non-characteristics $\tilde{\Gamma}_{2}, \tilde{\Gamma}_{2}^{\prime}, \tilde{\gamma}_{2}, \tilde{\gamma}_{2}^{\prime}, \tilde{\Delta}_{1}, \tilde{\Delta}_{1}^{\prime}, \tilde{\delta}_{1}, \tilde{\delta}_{1}^{\prime}:$

$$
\begin{aligned}
& \tilde{\Gamma}_{2}: \sqrt{M(x)} d x \geq \sqrt{-K(y)} d y>0 ; \quad \tilde{\Delta}_{1}^{\prime}: \sqrt{M(x)} d x \leq \sqrt{-K(y)} d y<0 ; \\
& \tilde{\Gamma}_{2}^{\prime}: \sqrt{M(x)} d x \leq-\sqrt{-K(y)} d y \leq 0 ; \quad \tilde{\Delta}_{1}: \sqrt{M(x)} d x \geq-\sqrt{-K(-y)} d y>0 ; \\
& \tilde{\gamma}_{2}: 0 \geq \sqrt{-M(x)} d x \geq \sqrt{K(y)} d y ; \quad \tilde{\delta}_{1}^{\prime}: 0 \leq \sqrt{-M(x)} d x \leq \sqrt{K(y)} d y ; \\
& \tilde{\gamma}_{2}^{\prime}: 0 \geq \sqrt{-M(x)} d x \geq-\sqrt{K(y)} d y ; \quad \tilde{\delta}_{1}: 0 \leq \sqrt{-M(x)} d x \leq-\sqrt{K(y)} d y,
\end{aligned}
$$

or

$$
\begin{aligned}
& \tilde{\Gamma}_{2} \cup \tilde{\Delta}_{1}^{\prime}: 0 \leq \frac{d y}{d x}\left(=\frac{\nu_{1}}{-\nu_{2}}\right) \leq \frac{\sqrt{M(x)}}{\sqrt{-K(y)}} ; \quad \tilde{\Gamma}_{2}^{\prime} \cup \tilde{\Delta}_{1}: 0 \geq \frac{d y}{d x} \geq-\frac{\sqrt{M(x)}}{\sqrt{-K(y)}} \\
& \tilde{\gamma}_{2} \cup \tilde{\delta}_{1}^{\prime}: \frac{d y}{d x} \geq \frac{\sqrt{-M(x)}}{\sqrt{K(y)}} ; \tilde{\gamma}_{2}^{\prime} \cup \tilde{\delta}_{1}: \frac{d y}{d x} \leq-\frac{\sqrt{-M(x)}}{\sqrt{K(y)}}
\end{aligned}
$$

satisfying the non-characteristic relation $K(y)(d y)^{2}+M(x)(d x)^{2}>0$, and intersecting characteristics $\Gamma_{1}, \Gamma_{1}^{\prime}, \gamma_{1}, \gamma_{1}^{\prime}, \Delta_{2}, \Delta_{2}^{\prime}, \delta_{2}, \delta_{2}^{\prime}$, only once. Let us consider the intersection points of the hyperbolic characteristics: $\tilde{\Gamma}_{2} \cap \tilde{\Gamma}_{2}^{\prime}=\left\{\tilde{\mathrm{P}}_{2}\right\}$, where $\tilde{\mathrm{P}}_{2}=\left(\tilde{x}_{2}, 1 / 2\right), 1<\tilde{x}_{2}<3 / 2 ; \tilde{\Delta}_{1} \cap \tilde{\Delta}_{1}^{\prime}=\left\{\tilde{\mathrm{P}}_{1}^{\prime}\right\}$, where $\tilde{\mathrm{P}}_{1}^{\prime}=\left(\tilde{x}_{1}^{\prime}, 1 / 2\right),-5 / 2<\tilde{x}_{1}^{\prime}<-2 ; \tilde{\gamma}_{2} \cap \tilde{\gamma}_{2}^{\prime}=\left\{\tilde{Q}_{2}\right\}$, where $\tilde{Q}_{2}=\left(-1 / 2, \tilde{y}_{2}\right), 2<\tilde{y}_{2}<5 / 2$; 
$\tilde{\delta}_{1} \cap \tilde{\delta}_{1}^{\prime}=\left\{\tilde{Q}_{1}^{\prime}\right\}$ where $\tilde{Q}_{1}^{\prime}=\left(-1 / 2, \tilde{y}_{1}^{\prime}\right),-3 / 2<\tilde{y}_{1}^{\prime}<-1$. Let the right hyperbolic domain $\tilde{G}_{2} \subset G_{2}=\{(x, y) \in D: 0<x<2,0<y<1\}$ with boundary

$$
\partial \tilde{G}_{2}=\left(O_{1} B_{1}\right) \cup\left(O_{2} B_{2}\right) \cup\left(\Gamma_{1} \cup \Gamma_{1}^{\prime}\right) \cup\left(\tilde{\Gamma}_{2} \cup \tilde{\Gamma}_{2}^{\prime}\right) .
$$

Let the upper hyperbolic domain $\tilde{G}_{2}^{\prime} \subset G_{2}^{\prime}=\{(x, y) \in D:-1<x<0,1<y<3\}$ with boundary

$$
\partial \tilde{G}_{2}^{\prime}=\left(O_{1} Z_{1}\right) \cup\left(O_{1}^{\prime} E_{1}\right) \cup\left(\gamma_{1} \cup \gamma_{1}^{\prime}\right) \cup\left(\tilde{\gamma}_{2} \cup \tilde{\gamma}_{2}^{\prime}\right) .
$$

Let the left hyperbolic domain $\tilde{G}_{2}^{\prime \prime} \subset G_{2}^{\prime \prime}=\{(x, y) \in D:-3<x<-1,0<y<1\}$ with boundary

$$
\partial \tilde{G}_{2}^{\prime \prime}=\left(O_{1}^{\prime} A_{1}\right) \cup\left(O_{2}^{\prime} A_{2}\right) \cup\left(\tilde{\Delta}_{1} \cup \tilde{\Delta}_{1}^{\prime}\right) \cup\left(\Delta_{2} \cup \Delta_{2}^{\prime}\right) .
$$

Let the lower hyperbolic domain $\tilde{G}_{2}^{\prime \prime \prime} \subset G_{2}^{\prime \prime \prime}=\{(x, y) \in D:-1<x<0,-2<y<0\}$ with boundary

$$
\partial \tilde{G}_{2}^{\prime \prime \prime}=\left(O_{2} Z_{2}\right) \cup\left(O_{2}^{\prime} E_{2}\right) \cup\left(\tilde{\delta}_{1} \cup \tilde{\delta}_{1}^{\prime}\right) \cup\left(\delta_{2} \cup \delta_{2}^{\prime}\right) .
$$

Assume boundary conditions on the above exterior boundary $\operatorname{Ext}(\tilde{D})$ :

$$
u=\left\{\begin{array}{ccccccc}
\varphi_{1}(s) & \text { on } & \Gamma_{0} & ; & \varphi_{2}(s) & \text { on } & \Gamma_{0}^{\prime} \\
\varphi_{3}(s) & \text { on } & \Gamma_{0}^{\prime \prime} & ; & \varphi_{4}(s) & \text { on } & \Gamma_{0}^{\prime \prime \prime} \\
\tilde{\psi}_{1}(x) & \text { on } & \tilde{\Gamma}_{2} & ; & \tilde{\psi}_{2}(x) & \text { on } & \tilde{\Gamma}_{2}^{\prime} \\
\tilde{\psi}_{3}(x) & \text { on } & \tilde{\gamma}_{2} & ; & \tilde{\psi}_{4}(x) & \text { on } & \tilde{\gamma}_{2}^{\prime} \\
\tilde{\psi}_{5}(x) & \text { on } & \tilde{\Delta}_{1} & ; & \tilde{\psi}_{6}(x) & \text { on } & \tilde{\Delta}_{1}^{\prime} \\
\tilde{\psi}_{7}(x) & \text { on } & \tilde{\delta}_{1} & ; & \tilde{\psi}_{8}(x) & \text { on } & \tilde{\delta}_{1}^{\prime}
\end{array}\right.
$$

with continuous prescribed values.

\section{The exterior Bitsadze-Lavrentjev problem: Frankl Case or Problem (BL-F):}

consists of finding a solution $u$ of the quaterelliptic -quaterhyperbolic equation (1.2) with eight parabolic lines in $\tilde{D}(\subset D)$ and which assumes continuous prescribed values (3.1).

Theorem 3.1. (UNIQUENESS THEOREM): Consider the quaterelliptic - quaterhyperbolic equation (1.2) with eight parabolic lines and the boundary condition (3.1). Assume the above mixed doubly connected domain $\tilde{D}(\subset D)$ and the following conditions:

$\left(R_{1}\right) \quad r \leq 0$ on the interior boundary $\operatorname{Int}(\tilde{D})(=\operatorname{Int}(D))$,

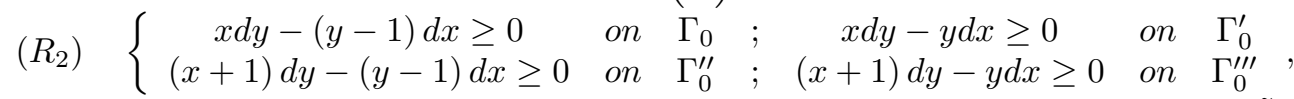

$\left(R_{3}\right)\left\{\begin{array}{ccccccc}2 r+x r_{x}+(y-1) r_{y} \leq 0 & \text { in } & G_{1} & ; & r+x r_{x} \leq 0 & \text { in } & \tilde{G}_{2} \\ 2 r+x r_{x}+y r_{y} \leq 0 & \text { in } & G_{1}^{\prime} & ; & r+(y-1) r_{y} \leq 0 & \text { in } & \tilde{G}_{2}^{\prime} \\ 2 r+(x+1) r_{x}+(y-1) r_{y} \leq 0 & \text { in } & G_{1}^{\prime \prime} & ; & r+(x+1) r_{x} \leq 0 & \text { in } & \tilde{G}_{2}^{\prime \prime} \\ 2 r+(x+1) r_{x}+y r_{y} \leq 0 & \text { in } & G_{1}^{\prime \prime \prime} & ; & r+y r_{y} \leq 0 & \text { in } & \tilde{G}_{2}^{\prime \prime \prime}\end{array}\right.$

$\left(R_{4}\right) \quad K=M=+1$, in $G_{1} \cup G_{1}^{\prime} \cup G_{1}^{\prime \prime} \cup G_{1}^{\prime \prime \prime}$, 
$\left(R_{5}\right) \quad\left\{\begin{array}{llll}K=-1 & , & M=+1 \quad \text { in } & \tilde{G}_{2} \cup \tilde{G}_{2}^{\prime \prime} \\ K=+1 & , & M=-1 & \text { in } \quad \tilde{G}_{2}^{\prime} \cup \tilde{G}_{2}^{\prime \prime \prime}\end{array}\right.$.

Let ()$_{x}=\partial() / \partial x,()^{\bullet}=d() / d x,()_{y}=\partial() / \partial y,()^{\prime}=d() / d y$, where $f=f(x, y)$ is continuous in $\tilde{D}(\subset D), r=r(x, y)$ is once-continuously differentiable in $\tilde{D}(\subset D), K=K(y)):=\operatorname{sgn}(y(y-$ $1)|k(y)|)$ is a discontinuous function of $y$, for $y \in\left[-k_{1}, k_{2}\right]:-k_{1}=\inf \{y:(x, y) \in \tilde{D}(\subset D)\}$ and $k_{2}=\sup \{y:(x, y) \in \tilde{D}(\subset D)\}$, and $M=M(x):=\operatorname{sgn}(x(x+1)|m(x)|)$ is discontinuous for $x \in\left[-m_{1}, m_{2}\right]:-m_{1}=\inf \{x:(x, y) \in \tilde{D}(\subset D)\}$ and $m_{2}=\sup \{x:(x, y) \in \tilde{D}(\subset D)\}$, such that $k=k(y) \neq 0 ; m=m(x) \neq 0$, everywhere in $D$, as well as

$$
K=\left\{\begin{array}{ccc}
+1 & \text { for } & \{y<0\} \cup\{y>1\} \\
0 & \text { for } & \{y=0\} \cup\{y=1\} \\
-1 & \text { for } & \{0<y<1\}
\end{array} \quad ; \quad M=\left\{\begin{array}{cccc}
+1 & \text { for } & \{x<-1\} \cup\{x>0\} \\
0 & \text { for } & \{x=0\} \cup\{x=-1\} \\
-1 & \text { for } & \{-1<x<0\}
\end{array} .\right.\right.
$$

Then Problem (BL-F) has at most one quasi-regular solution in $\tilde{D}(\subset D)$.

Proof. We apply the well-known energy integral method, and employ the above Bitsadze-Lavrentjev equation (1.2) and boundary condition (3.1). First, we assume two quasi-regular solutions $u_{1}, u_{2}$ of Problem (BL-F). Then we claim that $u=u_{1}-u_{2}=0$ holds in the domain $\tilde{D}(\subset D)$.

In fact, we investigate

$$
0=\tilde{J}=2\langle\tilde{l} u, L u\rangle_{0}=\iint_{D} 2 \tilde{l} u L u d x d y
$$

where $\tilde{l} u=\tilde{b}(x) u_{x}+\tilde{c}(y) u_{y}$, and $L u=L\left(u_{1}-u_{2}\right)=L u_{1}-L u_{2}=f-f=0$ in $\tilde{D}(\subset D)$, with choices

$$
\tilde{b}=\tilde{b}(x)=\left\{\begin{array}{ccc}
x & \text { in } & G_{1} \cup G_{1}^{\prime} \cup \tilde{G}_{2} \\
x+1 & \text { in } & G_{1}^{\prime \prime} \cup G_{1}^{\prime \prime} \cup \tilde{G}_{2}^{\prime \prime} \\
0 & \text { in } & \tilde{G}_{2}^{\prime} \cup \tilde{G}_{2}^{\prime \prime \prime}
\end{array}\right.
$$

and

$$
\tilde{c}=\tilde{c}(y)=\left\{\begin{array}{ccc}
y & \text { in } & G_{1}^{\prime} \cup G_{1}^{\prime \prime \prime} \cup \tilde{G}_{2}^{\prime \prime \prime} \\
y-1 & \text { in } & G_{1} \cup G_{1}^{\prime \prime} \cup \tilde{G}_{2}^{\prime} \\
0 & \text { in } & \tilde{G}_{2} \cup \tilde{G}_{2}^{\prime \prime}
\end{array}\right.
$$

The rest of the proof is similar to the proof of the uniqueness theorem 2.2 .

Q.E.D.

For the exterior Tricomi problem, except clearly proving in additional that the following condition holds on the non-characteristic hyperbolic exterior boundary

$$
\begin{gathered}
\operatorname{ExtHn}(\tilde{D})=\left(\tilde{\Gamma}_{2} \cup \tilde{\Gamma}_{2}^{\prime}\right) \cup\left(\tilde{\gamma}_{2} \cup \tilde{\gamma}_{2}^{\prime}\right) \cup\left(\tilde{\Delta}_{1} \cup \tilde{\Delta}_{1}^{\prime}\right) \cup\left(\tilde{\delta}_{1} \cup \tilde{\delta}_{1}^{\prime}\right): \\
0<\tilde{b} \nu_{1}+\tilde{c} \nu_{2}=\left\{\begin{array}{ccc}
x \nu_{1} & \text { on } & \tilde{\Gamma}_{2} \cup \tilde{\Gamma}_{2}^{\prime} \\
(y-1) \nu_{2} & \text { on } & \tilde{\gamma}_{2} \cup \tilde{\gamma}_{2}^{\prime} \\
(x+1) \nu_{1} & \text { on } & \tilde{\Delta}_{1} \cup \tilde{\Delta}_{1}^{\prime} \\
y \nu_{2} & \text { on } & \tilde{\delta}_{1} \cup \tilde{\delta}_{1}^{\prime}
\end{array} .\right.
\end{gathered}
$$


We note that $0=J_{\tilde{D}}=I_{\tilde{D}}+I_{\partial \tilde{D}} ; I_{\tilde{D}} \geq 0 ; I_{\partial \tilde{D}} \geq 0$. In fact,

$$
\begin{aligned}
I_{\tilde{D}}= & -\iint_{G_{1}}\left(2 r+x r_{x}+(y-1) r_{y}\right) u^{2} d x d y-\iint_{G_{1}^{\prime}}\left(2 r+x r_{x}+y r_{y}\right) u^{2} d x d y \\
& -\iint_{G_{1}^{\prime \prime}}\left(2 r+(x+1) r_{x}+(y-1) r_{y}\right) u^{2} d x d y \\
& -\iint_{G_{1}^{\prime \prime \prime}}\left(2 r+(x+1) r_{x}+y r_{y}\right) u^{2} d x d y+\iint_{\tilde{G}_{2} \cup \tilde{G}_{2}^{\prime} \cup \tilde{G}_{2}^{\prime \prime} \cup \tilde{G}_{2}^{\prime \prime \prime}}\left(u_{x}^{2}+u_{y}^{2}\right) d x d y \\
& -\iint_{\tilde{G}_{2}}\left(r+x r_{x}\right) u^{2} d x d y-\iint_{\tilde{G}_{2}^{\prime}}\left(r+(y-1) r_{y}\right) u^{2} d x d y \\
& -\iint_{\tilde{G}_{2}^{\prime \prime}}\left(r+(x+1) r_{x}\right) u^{2} d x d y-\iint_{\tilde{G}_{2}^{\prime \prime \prime}}\left(r+y r_{y}\right) u^{2} d x d y \geq 0 .
\end{aligned}
$$

Also

$$
\begin{aligned}
I_{\partial \tilde{D}}= & I_{\partial D}+\left[\left(I_{\tilde{\Gamma}_{2}}+I_{\tilde{\Gamma}_{2}^{\prime}}\right)+\left(I_{\tilde{\gamma}_{2}}+I_{\tilde{\gamma}_{2}^{\prime}}\right)+\left(I_{\tilde{\Delta}_{1}}+I_{\tilde{\Delta}_{1}^{\prime}}\right)+\left(I_{\tilde{\delta}_{1}}+I_{\tilde{\delta}_{1}^{\prime}}\right)\right] \\
= & \int_{\Gamma_{0}}\left(x \nu_{1}+(y-1) \nu_{2}\right)\left(\nu_{1}^{2}+\nu_{2}^{2}\right) N^{2} d s \\
& +\int_{\Gamma_{0}^{\prime}}\left(x \nu_{1}+y \nu_{2}\right)\left(\nu_{1}^{2}+\nu_{2}^{2}\right) N^{2} d s+\int_{\Gamma_{0}^{\prime \prime}}\left((x+1) \nu_{1}+(y-1) \nu_{2}\right)\left(\nu_{1}^{2}+\nu_{2}^{2}\right) N^{2} d s \\
& +\int_{\Gamma_{0}^{\prime \prime \prime}}\left((x+1) \nu_{1}+y \nu_{2}\right)\left(\nu_{1}^{2}+\nu_{2}^{2}\right) N^{2} d s+\int_{\Gamma_{1}} x\left[\left(u_{x}-u_{y}\right)^{2}-r u^{2}\right] d x \\
& -\int_{\Gamma_{1}^{\prime}} x\left[\left(u_{x}+u_{y}\right)^{2}-r u^{2}\right] d x+\int_{\gamma_{1}}(y-1)\left[\left(u_{x}-u_{y}\right)^{2}-r u^{2}\right] d x
\end{aligned}
$$




$$
\begin{aligned}
& +\int_{\gamma_{1}^{\prime}}(y-1)\left[\left(u_{x}+u_{y}\right)^{2}-r u^{2}\right] d x-\int_{\Delta_{2}}(x+1)\left[\left(u_{x}+u_{y}\right)^{2}-r u^{2}\right] d x \\
& +\int_{\Delta_{2}^{\prime}}(x+1)\left[\left(u_{x}-u_{y}\right)^{2}-r u^{2}\right] d x+\int_{\delta_{2}} y\left[\left(u_{x}+u_{y}\right)^{2}-r u^{2}\right] d x \\
& +\int_{\delta_{2}^{\prime}} y\left[\left(u_{x}-u_{y}\right)^{2}-r u^{2}\right] d x+\int_{\tilde{\Gamma}_{2} \cup \tilde{\Gamma}_{2}^{\prime}} x \nu_{1}\left(-\nu_{1}^{2}+\nu_{2}^{2}\right) N^{2} d s \\
& +\int_{\tilde{\gamma}_{2} \cup \tilde{\gamma}_{2}^{\prime}}(y-1) \nu_{2}\left(\nu_{1}^{2}-\nu_{2}^{2}\right) N^{2} d s+\int_{\tilde{\Delta}_{1} \cup \tilde{\Delta}_{1}^{\prime}}(x+1) \nu_{1}\left(-\nu_{1}^{2}+\nu_{2}^{2}\right) N^{2} d s \\
& +\int_{\tilde{\delta}_{1} \cup \tilde{\delta}_{1}^{\prime}} y \nu_{2}\left(\nu_{1}^{2}-\nu_{2}^{2}\right) N^{2} d s \geq 0,
\end{aligned}
$$

because of the following conditions:

$$
\left.x \nu_{1}\right|_{\tilde{\Gamma}_{2} \cup \tilde{\Gamma}_{2}^{\prime}} \geq 0 ;\left.(y-1) \nu_{2}\right|_{\tilde{\gamma}_{2} \cup \tilde{\gamma}_{2}^{\prime}} \geq 0 ;\left.(x+1) \nu_{1}\right|_{\tilde{\Delta}_{1} \cup \tilde{\Delta}_{1}^{\prime}} \geq 0 ;\left.y \nu_{2}\right|_{\tilde{\delta}_{1} \cup \tilde{\delta}_{1}^{\prime}} \geq 0
$$

as well as:

$$
\left.\left(-\nu_{1}^{2}+\nu_{2}^{2}\right)\right|_{\left(\tilde{\Gamma}_{2} \cup \tilde{\Gamma}_{2}^{\prime}\right) \cup\left(\tilde{\Delta}_{1} \cup \tilde{\Delta}_{1}^{\prime}\right)} \geq 0 \quad ;\left.\quad\left(\nu_{1}^{2}-\nu_{2}^{2}\right)\right|_{\left(\tilde{\gamma}_{2} \cup \tilde{\gamma}_{2}^{\prime}\right) \cup\left(\tilde{\delta}_{1} \cup \tilde{\delta}_{1}^{\prime}\right)} \geq 0 .
$$

We note that $\Gamma_{2}$ has an analogous equation as $\Gamma_{1}^{\prime}$, because $\Gamma_{2}$ is parallel to $\Gamma_{1}^{\prime}$.

On the non-characteristic $\tilde{\Gamma}_{2}: u=0$, one obtains

$$
I_{\tilde{\Gamma}_{2}}=-\int_{\tilde{\Gamma}_{2}} x\left[\left(u_{x}^{2}+u_{y}^{2}\right) d y+2 u_{x} u_{y} d x-r u^{2} d y\right] \geq 0,
$$

as due to $\left.u\right|_{\tilde{\Gamma}_{2}}=0: u_{x}=\nu_{1} N, u_{y}=\nu_{2} N, N=$ normalizing factor, such that

$$
\begin{aligned}
\left(u_{x}^{2}+u_{y}^{2}\right) d y+2 u_{x} u_{y} d x-r u^{2} d y & =\left[\left(\nu_{1}^{2}+\nu_{2}^{2}\right) \nu_{1}+2 \nu_{1} \nu_{2}\left(-\nu_{2}\right)-r .0\right] N^{2} d s \\
& =-\nu_{1}\left(-\nu_{1}^{2}+\nu_{2}^{2}\right) N^{2} d s \geq 0, \\
-\nu_{1}^{2}+\nu_{2}^{2} & =-(d y / d s)^{2}+(-d x / d s)^{2}=(d x-d y)(d x+d y) /(d s)^{2} \geq 0,
\end{aligned}
$$

where $0<d y \leq d x$ on $\tilde{\Gamma}_{2}$, and $\left.\nu_{1}\right|_{\tilde{\Gamma}_{2}}<0$.

Similarly on the following non-characteristics: $\tilde{\Gamma}_{2}^{\prime}, \tilde{\Delta}_{1}, \tilde{\Delta}_{1}^{\prime}: u=0$,

where $\left.x \nu_{1}\right|_{\tilde{\Gamma}_{2} \cup \tilde{\Gamma}_{2}^{\prime}} \geq 0 ;\left.(x+1) \nu_{1}\right|_{\tilde{\Delta}_{1} \cup \tilde{\Delta}_{1}^{\prime}} \geq 0$,

as well as: $\left.\left(-\nu_{1}^{2}+\nu_{2}^{2}\right)\right|_{\left(\tilde{\Gamma}_{2} \cup \tilde{\Gamma}_{2}^{\prime}\right) \cup\left(\tilde{\Delta}_{1} \cup \tilde{\Delta}_{1}^{\prime}\right)} \geq 0$.

On the other hand, on the non-characteristic $\tilde{\gamma}_{2}: u=0$, we find

$$
I_{\tilde{\gamma}_{2}}=\int_{\tilde{\gamma}_{2}}(y-1)\left[\left(u_{x}^{2}+u_{y}^{2}\right) d x+2 u_{x} u_{y} d y-r u^{2} d x\right] \geq 0,
$$


as due to $\left.u\right|_{\tilde{\gamma}_{2}}=0: u_{x}=\nu_{1} N, u_{y}=\nu_{2} N, N=$ normalizingfactor, such that

$$
\begin{gathered}
\left(u_{x}^{2}+u_{y}^{2}\right) d x+2 u_{x} u_{y} d y-r u^{2} d x= \\
=\nu_{2}\left(\nu_{1}^{2}-\nu_{2}^{2}\right) N^{2} d s \geq 0, \\
\nu_{1}^{2}-\nu_{2}^{2}=(d y / d s)^{2}-(-d x / d s)^{2}=(d y-d x)(d y+d x) /(d s)^{2} \geq 0,
\end{gathered}
$$

where $d y \leq d x<0$ on $\tilde{\gamma}_{2}$, and $\left.\nu_{2}\right|_{\tilde{\gamma}_{2}}>0$.

Similarly on the following non-characteristics: $\tilde{\gamma}_{2}^{\prime}, \tilde{\delta}_{1}, \tilde{\delta}_{1}^{\prime}: u=0$,

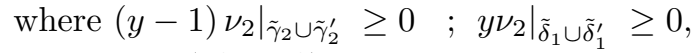
as well as: $\left.\left(\nu_{1}^{2}-\nu_{2}^{2}\right)\right|_{\left(\tilde{\gamma}_{2} \cup \tilde{\gamma}_{2}^{\prime}\right) \cup\left(\tilde{\delta}_{1} \cup \tilde{\delta}_{1}^{\prime}\right)} \geq 0$.

\section{Open problems}

4.1. Extend "quasi-regularity" of solutions to "regularity" by fixing singularities at the following twelve "singular" points:

$$
\begin{gathered}
O_{1}=(0,1), O_{1}^{\prime}=(-1,1), O_{2}=(0,0), O_{2}^{\prime}=(-1,0) ; \\
A_{1}=(-3,1), B_{1}=(2,1), A_{2}=(-3,0), B_{2}=(2,0) ; \\
E_{1}=(-1,3), Z_{1}=(0,3), E_{2}=(-1,-2), Z_{2}=(0,-2) .
\end{gathered}
$$

Then find the formula for these regular solutions.

4.2. Investigate the exterior Bitsadze-Lavrentjev problem in a multiply connected mixed domain.

4.3. Establish "well-posedness" of solutions for the 3D exterior Bitsadze-Lavrentjev problem , in the sense that there is at most one quasi-regular solution and a weak solution exists.

4.4. Solve the $\mathrm{n}$ - dimensional Bitsadze-Lavrentjev problem in a multiply connected mixed domain.

4.5. Establish the extremum principle for the exterior Bitsadze-Lavrentjev problem : "A solution of the exterior Bitsadze-Lavrentjev problem , vanishing on the exterior boundary of the considered mixed domain, achieves neither a positive maximum nor a negative minimum on open arcs of the type-degeneracy curves."

4.6. Solve the Bitsadze-Lavrentjev problem for Partial Differential Equations of second order:

4.6.1. $\operatorname{sgn}\left(x^{2}+y^{2}-1\right) u_{x x}+u_{y y}+r(x, y) u=f(x, y)$;

4.6.2. $\operatorname{sgn}\left(y^{3}-y\right) u_{x x}+\operatorname{sgn}\left(x^{3}-x\right) u_{y y}+r(x, y) u=f(x, y)$;

4.6.3. $\operatorname{sgn}\left(x^{2 / 3}+y^{2 / 3}-1\right) u_{x x}+u_{y y}+r(x, y) u=f(x, y)$;

4.6.4. $\operatorname{sgn}\left(\left(y-x^{m}\right)\left(y-x^{n}\right)\right) u_{x x}+u_{y y}+r(x, y) u=f(x, y)$;

4.6.5. $\operatorname{sgn}\left(y-x^{n}\right) u_{x x}+\operatorname{sgn}\left(x-y^{m}\right) u_{y y}+r(x, y) u=f(x, y)$;

4.6.6. $\operatorname{sgn}\left(y^{k}-x^{m} \pm x^{n}\right) u_{x x}+\operatorname{sgn}\left(x^{k}-y^{m} \pm y^{n}\right) u_{y y}+r(x, y) u=f(x, y)$;

4.6.7. $\operatorname{sgn}\left(y^{m}\left(y-x^{n}\right)\right) u_{x x}+\operatorname{sgn}\left(x^{m}\left(x-y^{n}\right)\right) u_{y y}+r(x, y) u=f(x, y)$;

4.6.8. $\operatorname{sgn}\left(\left(y-x^{m}\right)\left(y-x^{n}\right)\right) u_{x x}+\operatorname{sgn}\left(\left(x-y^{\alpha}\right)\left(x-y^{\beta}\right)\right) u_{y y}+r u=f(x, y)$. 
4.7. Solve the Bitsadze-Lavrentjev problem for PDE of fourth order:

$$
\left(\operatorname{sgn}\left(y-x^{l}\right) \frac{\partial^{2}}{\partial x^{2}}+\operatorname{sgn}\left(x-y^{n}\right) \frac{\partial^{2}}{\partial y^{2}}+r\right)^{2} u=f .
$$

4.8. Solve the 3 - dimensional Bitsadze-Lavrentjev problem for mixed type PDE of second order:

$$
\operatorname{sgn}(z)\left(u_{x x} \pm u_{y y}\right)+\operatorname{sgn}(x y) u_{z z}+r u=f .
$$

Acknowledgment: I greatly thank Dr. M. Arunkumar for the LaTEX typing of this long research paper.

\section{References}

[1] G. Barantsev, On singularities of the Tricomi problem solution by the Fourier method, Teubner-Texte zur Mathematik (Ed. J. M. Rassias), Vol. 90, Leipzig, 1986, pp. 47-54.

[2] G. Fichera, Francesco Giacomo Tricomi, Teubner-Texte zur Mathematik (Ed. J. M. Rassias), Vol. 79, Leipzig, 1985, pp. 6-31.

[3] F. I. Frankl, On the problems of Chaplygin for mixed subsonic and supersonic flows, Izv. Akad. Nauk SSSR Ser. Mat. 9 (1945), 121-143.

[4] M. Kracht and E. Kreyszig, The Tricomi equation and transition problems, Teubner-Texte zur Mathematik (Ed. J. M. Rassias), Vol. 90, Leipzig, 1986, pp. 157-165.

[5] E. Kreyszig, Introductory Functional Analysis with Applications, Wiley, New York, 1989.

[6] E. Kreyszig, Banach spaces in Bergman Operator Theory, World Scientific (Ed. J. M. Rassias), Singapore, 1994, pp. 155-165.

[7] M. H. Protter, Uniqueness theorems for the Tricomi problem, I, II, J.Rat. Mech. Anal. 2 (1953), 107-114; 4(1955), 721-732.

[8] J. M. Rassias, Mixed type partial differential equations in $\mathbb{R}^{n}$, Ph.D. dissertation, U.C. Berkeley, 1977.

[9] J. M. Rassias, A maximum principle in $\mathbb{R}^{n+1}$, J. Math. Anal. \& Appl., Acad. Press, New York, 85, (1982), 106-113.

[10] J. M. Rassias, On the Tricomi problem with two parabolic lines of degeneracy, Bull. Inst. Math., Acad. Sinica, 12 (1983), 62-67.

[11] J. M. Rassias, Lecture Notes on Mixed Type Partial Differential Equations, World Scientific, Singapore, 1990.

[12] J. M. Rassias, On the Well-posed Tricomi problem in $\mathbb{R}^{2}$, Discuss. Math., 12 (1992), 85-93. 
[13] J. M. Rassias, Uniqueness of Quasi-regular Solutions for a parabolic elliptic-hyperbolic Tricomi problem, Bull. Inst. Math., Acad. Sinica, 25 (1997), 277-287.

[14] J. M. Rassias, Advances in Equations and Inequalities, Hadronic Press, Inc., Palm Harbor, FL., U.S.A., 1999.

[15] J. M. Rassias, Existence of Weak Solutions for a parabolic elliptic-hyperbolic Tricomi problem, Tsukuba J. Math., 23(1999), 37-54.

[16] J. M. Rassias, Uniqueness of Quasi-Regular Solutions for a Bi-Parabolic Elliptic Bi-hyperbolic Tricomi Problem, Complex Variables and Elliptic Equations, 47(8) (2002), 707-718.

[17] J. M. Rassias and G. C. Wen, Solvability of the Oblique Derivative Problem for Second Order Equations of Mixed Type with Nonsmooth Degenerate Curve, Intern. J. Appl. Math. Stat., 8(M07)(2007), 96-111.

[18] R. I. Semerdjieva, Uniqueness of regular solutions for a class of non-linear degenerating hyperbolic equations, Math. Balk., 7 (1993), 277-283.

[19] F. G. Tricomi, Sulle equazioni lineari alle parziali di $2^{0}$ ordine di tipo misto, Atti Accad. Naz. Lincei, 14 (1923), 133-247.

[20] G. C. Wen, The Exterior Tricomi Problem for Generalized Mixed Equations with Parabolic Degeneracy, Acta Math. Sinica, 22(5)(2006), 1385-1398.

[21] G. C. Wen, Oblique Derivative Problems for General Chaplygin-Rassias Equations with Nonsmooth Degenerate Line in Mixed Domains, Science in China, Series A: Mathematics, $51(1)(2008), 5-36$.

[22] G. C. Wen, The Tricomi and Frankl Problems for Generalized Chaplygin Equations in Multiply Connected Domains, Acta Math. Sin., 24(11), 1759-1774.

[23] G. C. Wen, Oblique Derivative Problems for Generalized Rassias Equations of Mixed Type with Several Characteristic Boundaries, Electr. J. Diff. Equations, 2009(65)(2009), 1-16.

[24] G. C. Wen, Elliptic, Hyperbolic and Mixed Complex Equations with Parabolic Degeneracy / Including Tricomi-Bers and Tricomi-Frankl-Rassias Problems], World Scientific Co. Pte. Ltd., Singapore : Peking University, Series in Mathematics - Vol. 4 , 2008, 1-439.

[25] G. C. Wen and H. Begehr, Boundary Value Problems for Elliptic Equations and Systems, Longman Scientific and Technical Company, Harlow,1990.

[26] G. C. Wen, D. C. Chen and X. Cheng, General Tricomi-Rassias Problem and Oblique Derivative Problem for generalized Chaplygin Equation, J. Math. Anal. 333 (2007), 679-694.

[27] G. C. Wen and D. C. Chen, Discontinuous Riemann-Hilbert Problems for Degenerate Elliptic Complex Equations of First Order, Complex Variables, 50(2005), 707-718.

[28] G. C. Wen and Z. T. Ma, Discontinuous Oblique Derivative Problem for Second Order Equations of Mixed Type in General Domains, Complex Variables, 48(2)(2003), 119-130. 
[29] M. A. Lavrentjev and A. V. Bitsadze, On the Problem of the Equations of the Mixed Type, Dokl. Akad. Nauk. SSSR 70 (3) (1950), 373-376.

[30] A. V. Bitsadze, On the Problem of Equations of the Mixed Type, Doctoral Thesis: Library of the Mat. Inst. Akad. Nauk. SSSR (1951).

[31] G. C. Wen (Chief Editor), Boundary Value Problems, Integral Equations and Related Topics, Proc. of 3rd Int. Conf., 2011, World Sci. Publ. Co. Pte. Ltd.

[32] J. M. Rassias, The Exterior Tricomi and Frankl Problems for Quaterelliptic-Quaterhyperbolic Equations with Eight Parabolic Lines, European J. Pure and Appl. Math., 4(2)(2011), 186208.

[33] J. M. Rassias, Uniqueness of Solutions for the Exterior Quaterelliptic-Quaterhyperbolic Tricomi Problem with Eight Parabolic Lines, In: Boundary Value Problems, Integral Equations and Related Topics, Proceedings of the Third International Conference, World Sci. Publ. Co. Pte. Ltd., 2011(1), 58-71.

[34] D. Amanov and J. M. Rassias, Boundary value problems for the higher order generalized mixed-parabolic equation with fractional derivatives, Contemporary Analysis and Applied Mathematics, 2(2) (2014), 198-211. 